TRANSACTIONS OF THE

AMERICAN MATHEMATICAL SOCIETY

Volume 359, Number 9, September 2007, Pages 4467-4488

S 0002-9947(07)04187-6

Article electronically published on April 16, 2007

\title{
STRONGLY SINGULAR CONVOLUTION OPERATORS ON THE HEISENBERG GROUP
}

NEIL LYALL

\begin{abstract}
We consider the $L^{2}$ mapping properties of a model class of strongly singular integral operators on the Heisenberg group $\mathbf{H}^{n}$; these are convolution operators on $\mathbf{H}^{n}$ whose kernels are too singular at the origin to be of CalderónZygmund type. This strong singularity is compensated for by introducing a suitably large oscillation.

Our results are obtained by utilizing the group Fourier transform and uniform asymptotic forms for Laguerre functions due to Erdélyi.
\end{abstract}

\section{Overture: Strongly SINGULAR CONVOLUtion OPERATORS ON $\mathbf{R}^{d}$}

These are convolution operators whose kernels are too singular at the origin to be of Calderón-Zygmund type. We choose to compensate for this strong singularity by introducing a suitably large oscillation. More precisely these are the operators given formally by

$$
S f=f * K,
$$

where $K$ is a distribution on $\mathbf{R}^{d}$ that away from the origin agrees with the function

$$
K(x)=|x|^{-d-\alpha} e^{i|x|^{-\beta}} \chi(|x|),
$$

where $\beta>0$ and $\chi$ is smooth cut off function which equals one near the origin 1 Operators of this type were first studied by Hirschman [7] in the case $d=1$ and then in higher dimensions by Wainger [12, Fefferman 3], and Fefferman and Stein 44. What is of interest here is the precise relationship between the size of the singularity and that of the required oscillation in order for $S$ to extend to a bounded operator on $L^{2}\left(\mathbf{R}^{d}\right)$.

Theorem A. $S$ extends to a bounded operator on $L^{2}\left(\mathbf{R}^{d}\right)$ if and only if $\alpha \leq \frac{d \beta}{2}$.

Sketch of proof. Since $S$ is translation invariant it may be realized as a Fourier multiplier,

$$
\widehat{S f}(\xi)=\widehat{f}(\xi) \cdot m(\xi),
$$

where ${ }^{\wedge}$ denotes the Fourier transform and $m=\widehat{K}$, the fact that $K$ is a compactly supported distribution ensures that $m(\xi)$ is a function. Plancherel's theorem then implies that

$$
\|S f\|_{L^{2}\left(\mathbf{R}^{d}\right)} \leq A\|f\|_{L^{2}\left(\mathbf{R}^{d}\right)} \text { if and only if } \quad|m(\xi)| \leq A, \text { uniformly in } \xi .
$$

Received by the editors November 12, 2004 and, in revised form, October 10, 2005.

2000 Mathematics Subject Classification. Primary 42B20, 43A80.

1 The distribution-valued function $\alpha \mapsto K$, initially defined for $\operatorname{Re} \alpha<0$, continues analytically to all of $\mathbf{C}$.

(C)2007 American Mathematical Society Reverts to public domain 28 years from publication 
Since $K$ is also radial, i.e. $K(x)=K_{0}(x)$ for some function $K_{0}$, we have

$$
m(\xi)=(2 \pi)^{\frac{d}{2}} \int_{0}^{\infty} K_{0}(r) J_{\frac{d-2}{2}}(r|\xi|)(r|\xi|)^{\frac{2-d}{2}} r^{d-1} d r
$$

where $J_{\frac{d-2}{2}}$ is a Bessel function; see [10]. Using the well known asymptotics of Bessel functions it is then straightforward to show that $m(\xi)$ remains bounded for small $|\xi|$ and that for large $|\xi|$,

$$
m(\xi)=c_{1}|\xi|^{\frac{\alpha-\frac{d \beta}{2}}{\beta+1}} e^{i c_{2}|\xi|^{\frac{\beta}{\beta+1}}}+O\left(|\xi|^{\frac{\alpha-\frac{d+1}{2} \beta}{\beta+1}}\right) .
$$

Remark. One can establish Theorem A independent of Fourier transform methods; see $[8$.

This article is devoted to the study of analogous operators on the Heisenberg group.

\section{INTRODUCTION}

The Heisenberg group $\mathbf{H}^{n}$ is of course $\mathbf{C}^{n} \times \mathbf{R}$ endowed with the group law

$$
[z, t] \cdot[w, s]=\left[z+w, t+s+\frac{1}{2} \operatorname{Im}(z \cdot \bar{w})\right],
$$

with identity the origin and inverses given by $[z, t]^{-1}=[-z,-t]$. The following transformations are automorphisms of the group $\mathbf{H}^{n}$ :

- the nonisotropic dilations $[z, t] \mapsto \delta \circ[z, t]=\left[\delta z, \delta^{2} t\right]$, for all $\delta>0$;

- the rotations $[z, t] \mapsto[U z, t]$, with $U$ a unitary transformation of $\mathbf{C}^{n}$.

We define the "norm" function $\rho$ on $\mathbf{H}^{n}$ to be

$$
\rho(z, t)=\left(|z|^{4}+t^{2}\right)^{1 / 4},
$$

and note that it is smooth, invariant under the rotations defined above, and homogeneous with respect to the automorphic dilations. The usual Lebesgue measure $d z d t$ on $\mathbf{C}^{n} \times \mathbf{R}$ is the Haar measure for $\mathbf{H}^{n}$.

We define our strongly singular kernels on $\mathbf{H}^{n}$ to be the distributions $K$ that agree, for $(z, t) \neq(0,0)$, with the functions

$$
K(z, t)=\rho(z, t)^{-Q-\alpha} e^{i \rho(z, t)^{-\beta}} \chi(\rho(z, t)) \text { with } \beta>0,
$$

where $Q=2 n+2$ is the homogeneous dimension of $\mathbf{H}^{n}$ and $\chi$ is defined as before 2

Our main result is then the following.

Theorem 1.1. The operators $T$ on $\mathbf{H}^{n}$, formally given by $T f=f * K$, satisfy the following:

(i) If $\alpha \leq n \beta$, then $T$ extends to a bounded operator from $L^{2}\left(\mathbf{H}^{n}\right)$ to itself.

(ii) If $T$ is a bounded operator on $L^{2}\left(\mathbf{H}^{n}\right)$, then $\alpha \leq\left(n+\frac{1}{2}\right) \beta$.

Remark 1.2. There is a "gap of $\frac{1}{2}$ " between necessary and sufficient conditions. Note also that the necessary condition coincides with the Euclidean condition $\alpha \leq \frac{2 n+1}{2} \beta$.

2 As in the Eulidean case the distribution-valued function $\alpha \mapsto K$, initially defined for Re $\alpha<$ 0 , continues analytically to the entire complex plane. 


\section{Reduction to Laguerre transform estimates}

Let $\epsilon>0$ and set

$$
K^{\epsilon}(z, t)=e^{-\epsilon \rho(z, t)^{-\beta}} K(z, t),
$$

and for $f \in L^{2}\left(\mathbf{H}^{n}\right)$ we define

$$
T^{\epsilon} f(z, t)=f * K^{\epsilon}(z, t),
$$

where convolution is taken with respect to the group structure on $\mathbf{H}^{n}$. It is then easy to see that if, for fixed $\epsilon>0$, we integrate the function $K^{\epsilon}$ by parts $N$ times and take the limit as $\epsilon \rightarrow 0$, then this must agree with the unique analytic continuation of $K$ to the half plane $\operatorname{Re}(\alpha)<N \beta$. It then follows that for $f \in \mathcal{S}\left(\mathbf{H}^{n}\right)$,

$$
T f(z, t)=\lim _{\epsilon \rightarrow 0} T^{\epsilon} f(z, t) .
$$

We shall therefore, in the following, content ourselves with studying the operator $T^{\epsilon}$.

2.1. Group Fourier transform. Our operator $T^{\epsilon}$ on $\mathbf{H}^{n}$ can be realized on the "transform" side, via the group Fourier transform, as a multiplication of operators,

$$
\widehat{T^{\epsilon} f}(\lambda)=\widehat{f}(\lambda) \cdot M(\lambda),
$$

where $M(\lambda)$ is the group Fourier transform of $K^{\epsilon}$. Recall that, for each $\lambda \neq 0$, $M(\lambda)$ is an operator on the Hilbert space $L^{2}\left(\mathbf{R}^{n}\right)$ given by

$$
M(\lambda) \varphi(\xi)=\int_{\mathbf{H}^{n}} K^{\epsilon}(z, t) \pi_{\lambda}(z, t) \varphi(\xi) d z d t,
$$

where the $\pi_{\lambda}$, given for $z=x+i y$ by

$$
\pi_{\lambda}(z, t) \varphi(\xi)=e^{i \lambda\left(x \cdot \xi+\frac{1}{2} x \cdot y+t\right)} \varphi(\xi+y),
$$

are the infinite dimensional irreducible unitary representations of $\mathbf{H}^{n}$, the so called Schrödinger representations. It then follows from Plancherel's theorem for the group Fourier transform that

$\left\|T^{\epsilon} f\right\|_{L^{2}\left(\mathbf{H}^{n}\right)} \leq A\|f\|_{L^{2}\left(\mathbf{H}^{n}\right)}$ if and only if $\quad\|M(\lambda)\|_{O p} \leq A$ uniformly over $\lambda \neq 0$.

Now since $K^{\epsilon}$ were chosen radial on $\mathbf{H}^{n}$, i.e. $K^{\epsilon}(z, t)=K_{0}^{\epsilon}(|z|, t)$ for some function $K_{0}^{\epsilon}$, then it is a well known result of Geller 6 that the operators $M(\lambda)$ are in fact, for each $\lambda \neq 0$, diagonal with respect to a (rescaled) Hermite basis for $L^{2}\left(\mathbf{R}^{n}\right)$. More precisely

$$
M(\lambda)=C_{n}\left(\delta_{\mathbf{j}, \mathbf{k}} \mu(|\mathbf{k}|, \lambda)\right)_{\mathbf{j}, \mathbf{k} \in \mathbf{N}^{n}},
$$

where $C_{n}$ is a constant which depends only on the dimension and where the diagonal entries $\mu(|\mathbf{k}|, \lambda)$ can be expressed explicitly in terms a Laguerre transform. Denoting $\kappa=|\mathbf{k}|$ we in fact have

$$
\mu(\kappa, \lambda)=c_{\kappa}^{n-1} \int_{0}^{\infty} K_{0}^{\lambda}(s) \Lambda_{\kappa}^{n-1}\left(\frac{1}{2}|\lambda| s^{2}\right)\left(\frac{1}{2}|\lambda| s^{2}\right)^{\frac{1-n}{2}} s^{2 n-1} d s,
$$

where $c_{\kappa}^{\delta}=\left(\frac{\kappa !}{(\kappa+\delta) !}\right)^{1 / 2}, \Lambda_{\kappa}^{\delta}(x)$ is a Laguerre function 3 of type $\delta$, and $K^{\lambda}(z)=$ $\int_{\mathbf{R}} K(z, t) e^{i \lambda t} d t$.

\footnotetext{
3 For the definition and properties of Laguerre functions see 3.2
} 
It therefore follows that the operators $M(\lambda)$ are bounded on $L^{2}\left(\mathbf{R}^{n}\right)$ if and only if the diagonal scalars $\mu(\kappa, \lambda)$ are bounded uniformly in $\kappa$, and hence

$$
\left\|T^{\epsilon} f\right\|_{L^{2}\left(\mathbf{H}^{n}\right)} \leq A\|f\|_{L^{2}\left(\mathbf{H}^{n}\right)}
$$

if and only if $|\mu(\kappa, \lambda)| \lesssim A$ uniformly in $\kappa$ and $\lambda \neq 0$.

For more on the group Fourier transform and Laguerre functions see 9] and [11.

2.2. Main estimates. We have seen that matters therefore naturally reduce to the study of the 'Fourier transforms' $\mu(\kappa, \lambda)$. Making the change of variables

$$
(s, t) \mapsto\left(r \sin ^{\frac{1}{2}} \theta, r^{2} \cos \theta\right) \text { where } 0<r<\infty \text { and } 0 \leq \theta \leq \pi,
$$

it follows that $s^{4}+t^{2}=r^{4}, d s d t=r^{2} \sin ^{-\frac{1}{2}} \theta d r d \theta$, and hence that

$$
\begin{aligned}
\mu(\kappa, \lambda)= & c_{\kappa}^{n-1} \int_{0}^{\pi} \int_{0}^{\infty}(\sin \theta)^{n-1} \chi_{\epsilon}(r) r^{-1-\alpha} e^{i\left[r^{-\beta}+\lambda r^{2} \cos \theta\right]}\left(\frac{1}{2} \lambda r^{2} \sin \theta\right)^{\frac{1-n}{2}} \\
& \cdot \Lambda_{\kappa}^{n-1}\left(\frac{1}{2} \lambda r^{2} \sin \theta\right) d r d \theta,
\end{aligned}
$$

where $\chi_{\epsilon}(r)=e^{-\epsilon r^{-\beta}} \chi(r)$.

It is natural to consider the cases for bounded and unbounded $\kappa$ separately. We now fix $\kappa_{0}$ to be a large constant. Our main result for bounded $\kappa$ is the following.

Theorem 2.1. If $\kappa \leq \kappa_{0}$ and $|\lambda| \gg 1$, then we have the asymptotic expansion,

$$
\mu(\kappa, \lambda)=c_{1}|\lambda|^{\frac{\alpha-\left(n+\frac{1}{2}\right) \beta}{\beta+2}} e^{i c_{2}|\lambda|^{\frac{\beta}{\beta+2}}}+O\left(|\lambda|^{\frac{\alpha-(n+1-\varepsilon) \beta}{\beta+2}}\right) .
$$

For unbounded $\kappa$ we write $\mu(\kappa, \lambda)=\sum_{j=0}^{\infty} \mu_{j}(\kappa, \lambda)$, where

$\mu_{j}(\kappa, \lambda)=c_{\kappa}^{n-1} \int_{0}^{\pi} \int_{0}^{\infty}(\sin \theta)^{n-1} \vartheta\left(2^{j} r\right) \chi_{\epsilon}(r) r^{-1-\alpha} e^{i\left[r^{-\beta}+\lambda r^{2} \cos \theta\right]} x^{\frac{1-n}{2}} \Lambda_{\kappa}^{n-1}(x) d r d \theta$, with $x=\frac{1}{2} \lambda r^{2} \sin \theta$ and $\vartheta \in C_{0}^{\infty}(\mathbf{R})$ supported in $\left[\frac{1}{2}, 2\right]$ chosen such that $\sum_{j=0}^{\infty} \vartheta\left(2^{j} r\right)$ $=1$ for all $0 \leq r \leq 1$. Our main result for unbounded $\kappa$ is then the following.

Theorem 2.2. The following estimate holds uniformly for $\kappa \geq \kappa_{0}$ and $\lambda \neq 0$ :

$$
\left|\mu_{j}(\kappa, \lambda)\right| \leq C \min \left\{2^{j(\alpha-n \beta)},(1+|\lambda| \kappa)^{\frac{\alpha-n \beta}{2(\beta+1)}}\right\} .
$$

Remark 2.3. Theorem 2.2 alone only implies $|\mu(\kappa, \lambda)| \leq C \log (1+|\lambda| \kappa)(1+|\lambda| \kappa)^{\frac{\alpha-n \beta}{2(\beta+1)}}$ for $\kappa \geq \kappa_{0}$.

2.3. Proof of Theorem 1.1. Let us now see how Theorems 2.1 and 2.2 imply Theorem 1.1

It is clear that part (ii) of Theorem 1.1 follows immediately from (2). Furthermore, one easily obtains the estimate $|\mu(\kappa, \lambda)| \leq C$ for all $0<|\lambda| \leq \lambda_{0}$, for some fixed constant $\lambda_{0}$. It follows from the Laguerre function estimates in 33.2 that this estimate holds uniformly for all $\kappa$, however Theorem 2.2 shows us that for fixed $\lambda$ we in fact have decay in $\kappa$.

In order to prove Theorem 1.1 (i) we shall dyadically decompose our operator and use almost orthogonality. Recall the following version of Cotlar's Lemma,

Cotlar's Lemma. Suppose $\left\{T_{i}\right\}$ is a finite collection of bounded operators on $L^{2}$. If in addition these operators satisfy, for some $\delta>0$, the almost orthogonality condition

$$
\left\|T_{i}^{*} T_{j}\right\|_{O p}+\left\|T_{i} T_{j}^{*}\right\|_{O p} \leq C 2^{-\delta|i-j|},
$$


then

$$
\left\|\sum_{i} T_{i}\right\|_{O p} \leq A
$$

where the constant $A$ is independent of the number of these $T_{j}$.

For an elegant proof of this result see [9]. If we now define the dyadic operator $T_{j}=f * K_{j}$, where

$$
K_{j}(z, t)=\vartheta\left(2^{j} \rho(z, t)\right) K^{\epsilon}(z, t)
$$

it then follows that

$$
T^{\epsilon} f(z, t)=\sum_{j=0}^{\infty} T_{j} f(z, t) .
$$

Now the operators $T_{j}$ are bounded on $L^{2}\left(\mathbf{H}^{n}\right)$ if and only if

$$
\left|\mu_{j}(\kappa, \lambda)\right| \leq C
$$

uniformly in $\kappa$ and $\lambda \neq 0$. It is therefore an immediate consequence of Theorem 2.2 that the norms of $T_{j}$ are uniformly bounded whenever $\alpha \leq n \beta$. Theorem 1.1 now follows from an application of Cotlar's lemma (and a standard limiting argument) once we have verified that our operators $T_{j}$ are, in the following sense, almost orthogonal.

Lemma 2.4. If $\alpha=n \beta$, then $\left\|T_{i}^{*} T_{j}\right\|_{O p}+\left\|T_{i} T_{j}^{*}\right\|_{O p} \leq C 2^{-n \beta|i-j|}$.

Proof. This follows trivially whenever $|i-j| \leq 10$, since $\left\|T_{i}^{*} T_{j}\right\|_{O p} \leq\left\|T_{i}\right\|_{O p}\left\|T_{j}\right\|_{O p}$. We shall therefore, without loss of generality, assume that $j \geq i+10$. Now $T_{i}^{*} T_{j}$ has a kernel $L_{i j}(z, t)=K_{j} * \bar{K}_{i}\left([z, t]^{-1}\right)$, and the same operator norm as the operator with kernel the $L^{1}$ dilate of $L_{i j}$, namely

$$
\begin{aligned}
\widetilde{L}_{i j}\left([z, t]^{-1}\right)= & 2^{-j Q} L_{i j}\left(2^{-j} \circ[z, t]^{-1}\right) \\
= & 2^{-j Q} \int K_{j}(w, s) \bar{K}_{i}\left(\left(2^{-j} \circ[z, t]\right) \cdot[w, s]\right) d w d s \\
= & 2^{-j 2 Q} \int K_{j}\left(2^{-j} w, 2^{-2 j} s\right) \bar{K}_{i}\left(\left[2^{-j} z, 2^{-2 j} t\right] \cdot\left[2^{-j} w, 2^{-2 j} s\right]\right) d w d s \\
= & \left.2^{j 2 \alpha} \int_{\substack{\rho(w, s) \sim 1\\
}} \rho(w, s)^{-Q-\alpha} \rho([z, t] \cdot[w, s])^{-Q-\alpha} \cdot[w, s]\right) \sim 2^{j-i} \\
& \cdot e^{i 2^{j \beta}\left[\rho(w, s)^{-\beta}-\rho([z, t] \cdot[w, s])^{-\beta}\right]} d w d s .
\end{aligned}
$$

We trivially obtain the estimate $\left|\widetilde{L}_{i j}\left([z, t]^{-1}\right)\right| \leq C 2^{j 2 \alpha} 2^{(i-j)(Q+\alpha)}$, while integration by parts $2 n$ times gives the favorable estimate

$$
\left|\widetilde{L}_{i j}\left([z, t]^{-1}\right)\right| \leq C 2^{j(2 \alpha-2 n \beta)} 2^{(i-j)(Q+\alpha)}=2^{(i-j)(Q+\alpha)} .
$$

This of course implies that $\left\|\widetilde{L}_{i j}\right\|_{L^{1}\left(\mathbf{H}^{n}\right)} \leq C 2^{(i-j) \alpha}$.

As we have reduced matters to proving Theorem 2.1 and Theorem 2.2 , we present these arguments in Sections 4 and 5 respectively. As with the Euclidean case the asymptotics of special functions, in this case Laguerre polynomials, will be crucial. We discuss these expansions in Section 3 as well as stating some standard results for oscillatory integrals in one dimension that we shall require. 


\section{Preliminaries}

3.1. Oscillatory integrals. Here we present a very brief overview of the theory of oscillatory integrals in one dimension. This gives an essentially complete description of the behavior of integrals of the form

$$
I(\lambda)=\int_{a}^{b} e^{i \lambda \Phi(x)} \Psi(x) d x, \quad \lambda>0,
$$

as $\lambda \rightarrow \infty$, where $\Phi$ and $\Psi$ are smooth functions. The behavior of $I(\lambda)$ is governed by three basic principles: localization, scaling, and asymptotics. We shall present these respective principles as three propositions: the first of these can be thought of as a principle of non-stationary phase, the second is one of van der Corput's lemmas, and the third is a formulation of the method of stationary phase; for proofs see [9] or [13].

Proposition 3.1. Suppose $\Psi$ has compact support in $(a, b)$ and $\Phi^{\prime}$ never vanishes; then for all $N \geq 0$ we have

$$
|I(\lambda)| \leq C_{N, \Phi, \Psi} \lambda^{-N}
$$

Proposition 3.2. Suppose $\Phi$ is real-valued and $\left|\Phi^{(k)}(x)\right| \geq 1$ for all $x \in(a, b)$. Then

$$
|I(\lambda)| \leq C_{k} \lambda^{-\frac{1}{k}}\left[|\Psi(b)|+\int_{a}^{b}\left|\Psi^{\prime}(x)\right| d x\right],
$$

whenever (i) $k=1$ and $\Phi^{\prime \prime}(x)$ has at most one zero, or (ii) $k \geq 2$.

Proposition 3.3. Suppose $\Phi$ is real-valued, $\Phi^{\prime}\left(x_{0}\right)=0$, while $\Phi^{\prime \prime}\left(x_{0}\right) \neq 0$. If $\Psi$ is supported in a sufficiently small neighborhood of $x_{0}$, then

$$
I(\lambda)=e^{i \lambda \Phi\left(x_{0}\right)} \sigma(\lambda),
$$

where $\sigma$ is a symbol of order $-\frac{1}{2}$, that is, $\left|\sigma^{(\ell)}(\lambda)\right| \leq c_{\ell}(1+\lambda)^{-\frac{1}{2}-\ell}$. The constant $c_{\ell}$ depends on the $C^{\ell+1}$ norms of $\Phi$ and $\Psi$ on the $\operatorname{supp} \Psi$, the size of this support, and on a lower bound for $\left|\Phi^{\prime \prime}\left(x_{0}\right)\right|$.

Example 3.4. The Bessel functions, defined for real $k>-\frac{1}{2}$ by the formula

$$
J_{k}(\lambda)=\left(\pi^{\frac{1}{2}} \Gamma\left(k+\frac{1}{2}\right)\right)^{-1}\left(\frac{\lambda}{2}\right)^{k} \int_{-1}^{1} e^{i \lambda t}\left(1-t^{2}\right)^{k-\frac{1}{2}} d t,
$$

are a model case for these oscillatory integrals, and using the Propositions above one can show that

$$
J_{k}(\lambda)=\sigma_{1}(\lambda) e^{i \lambda}+\sigma_{2}(\lambda) e^{-i \lambda}
$$

where $\sigma_{i}$ is a symbol of order $-\frac{1}{2}$ for $i=1,2$.

3.2. Asymptotic properties of Laguerre functions. Recall that Laguerre functions of type $\delta, \delta>-1$, form an orthonormal basis for $L^{2}\left(\mathbf{R}^{+}\right)$and are given by

$$
\Lambda_{\kappa}^{\delta}(x)=\left(\frac{\kappa !}{(\kappa+\delta) !}\right)^{1 / 2} L_{\kappa}^{\delta}(x) e^{-\frac{1}{2} x} x^{\frac{\delta}{2}},
$$

where $L_{\kappa}^{\delta}(x)=\sum_{j=0}^{\kappa}\left(\begin{array}{c}\kappa+\delta \\ \kappa-j\end{array}\right) \frac{(-x)^{j}}{j !}$ are the Laguerre polynomials of type $\delta$. 
The two asymptotic formulae below which hold uniformly in their respective ranges of validity (which overlap) are due to Erdélyi [2]; see also [5]. In what follows $\nu=4 \kappa+2 \delta+2$ and $N=\nu / 4$.

3.2.1. The Bessel asymptotic forms. Let $0 \leq x \leq b \nu, b<1$. Then for $\kappa \geq \kappa_{0}$,

$$
\Lambda_{\kappa}^{\delta}(x)=\left(\frac{(\delta+\kappa) !}{\kappa !}\right)^{\frac{1}{2}} 2^{\delta-\frac{1}{2}} \nu^{-\frac{\delta}{2}}\left(\frac{\nu}{x}\right)^{\frac{1}{2}}\left(\frac{\psi}{\psi^{\prime}}\right)^{\frac{1}{2}}\left\{J_{\delta}(\nu \psi)+O\left[\nu^{-1}\left(\frac{x}{\nu-x}\right)^{\frac{1}{2}} \widetilde{J}_{\delta}(\nu \psi)\right]\right\},
$$

and so

$$
\Lambda_{\kappa}^{\delta}(x)=C_{1}(\delta)\left(\frac{\nu}{x}\right)^{\frac{1}{2}}\left(\frac{\psi}{\psi^{\prime}}\right)^{\frac{1}{2}}\left\{J_{\delta}(\nu \psi)+O\left[\nu^{-1}\left(\frac{x}{\nu-x}\right)^{\frac{1}{2}} \widetilde{J}_{\delta}(\nu \psi)\right]\right\}
$$

where $C_{1}(\delta)$ is a constant independent of $\kappa, \psi=\psi(t)$ satisfies

$$
\psi^{\prime}(t)=\frac{1}{2}\left(\frac{1}{t}-1\right)^{\frac{1}{2}}
$$

and $t=\frac{x}{\nu}$. For $0 \leq t<1$,

$$
\psi(t)=\frac{1}{2}\left[\left(t-t^{2}\right)^{\frac{1}{2}}+\sin ^{-1} t^{\frac{1}{2}}\right]
$$

and

$$
\widetilde{J}_{\delta}(u)= \begin{cases}J_{\delta}(u) & \text { if } u \text { is sufficiently small } \\ \left(\left|J_{\delta}(u)\right|^{2}+\left|Y_{\delta}(u)\right|^{2}\right)^{\frac{1}{2}} & \text { otherwise }\end{cases}
$$

here $Y_{\delta}$ and $J_{\delta}$ are Bessel functions of order $\delta$.

Lemma 3.5. If $0 \leq t \leq \frac{1}{2}$, then $\frac{1}{2} t^{\frac{1}{2}} \leq \psi(t) \leq t^{\frac{1}{2}}$.

Proof. Let $f(t)=\left(t-t^{2}\right)^{\frac{1}{2}}+\sin ^{-1} t^{\frac{1}{2}}$, notice then that $f^{\prime}(t)=\left(\frac{1-t}{t}\right)^{\frac{1}{2}}$. Now if $0 \leq s \leq \frac{1}{2}$, we have $\frac{1}{2} s^{-\frac{1}{2}} \leq f^{\prime}(s) \leq s^{-\frac{1}{2}}$, and so

$$
\frac{1}{2} \int_{0}^{t} s^{-\frac{1}{2}} d s \leq \int_{0}^{t} f^{\prime}(s) d s \leq \int_{0}^{t} s^{-\frac{1}{2}} d s
$$

which implies $t^{\frac{1}{2}} \leq f(t) \leq 2 t^{\frac{1}{2}}$, since $f(0)=0$.

3.2.2. The Airy asymptotic forms. Let $0<a \nu \leq x, a>0$. Then for $\kappa \geq \kappa_{0}$,

$$
\Lambda_{\kappa}^{\delta}(x)=\frac{(-1)^{\kappa}}{(\kappa !(\delta+\kappa) !)^{\frac{1}{2}}} 2^{\frac{5}{6}} N^{N+\frac{1}{6}} e^{-N} x^{-\frac{1}{2}}\left(\frac{\pi}{-\phi^{\prime}}\right)^{\frac{1}{2}}\left\{\operatorname{Ai}\left(-\nu^{\frac{2}{3}} \phi\right)+O\left[x^{-1} \widetilde{\operatorname{Ai}}\left(-\nu^{\frac{2}{3}} \phi\right)\right]\right\},
$$

and so, using Stirling's formula

$$
\Lambda_{\kappa}^{\delta}(x)=C_{2}(\delta)(-1)^{\kappa} \nu^{\frac{1}{6}} x^{-\frac{1}{2}}\left(\frac{1}{-\phi^{\prime}}\right)^{\frac{1}{2}}\left\{\operatorname{Ai}\left(-\nu^{\frac{2}{3}} \phi\right)+O\left[x^{-1} \widetilde{\operatorname{Ai}}\left(-\nu^{\frac{2}{3}} \phi\right)\right]\right\}
$$

where $C_{2}(\delta)$ is a constant independent of $\kappa, \phi=\phi(t)$ satisfies

$$
[\phi(t)]^{\frac{1}{2}} \phi^{\prime}(t)=\frac{1}{2}\left(\frac{1}{t}-1\right)^{\frac{1}{2}}
$$

and again $t=\frac{x}{\nu}$. Now one can show

$$
\phi(t)=\left(\frac{3}{4}\right)^{\frac{2}{3}} \begin{cases}{\left[\cos ^{-1} t^{\frac{1}{2}}-\left(t-t^{2}\right)^{\frac{1}{2}}\right]^{\frac{2}{3}}} & \text { if } 0<t \leq 1, \\ -\left[\left(t-t^{2}\right)^{\frac{1}{2}}-\cosh ^{-1} t^{\frac{1}{2}}\right]^{\frac{2}{3}} & \text { if } t>1\end{cases}
$$


and

$$
\widetilde{\operatorname{Ai}}(z)= \begin{cases}\operatorname{Ai}(z) & \text { if } z \geq 0, \\ \left(|\operatorname{Ai}(z)|^{2}+|\operatorname{Bi}(z)|^{2}\right)^{\frac{1}{2}} & \text { if } z \leq 0\end{cases}
$$

here Ai and Bi are Airy integrals 4

Lemma 3.6. If $\frac{1}{2} \leq t \leq 1$, then $\frac{1}{2}(1-t) \leq \phi(t) \leq 1-t$.

Proof. Let $g(t)=\cos ^{-1} t^{\frac{1}{2}}-\left(t-t^{2}\right)^{\frac{1}{2}}$, notice then that $g^{\prime}(t)=-\left(\frac{1-t}{t}\right)^{\frac{1}{2}}$. Now if $\frac{1}{2} \leq s \leq 1$, we have $(1-s)^{\frac{1}{2}} \leq-g^{\prime}(s) \leq 2(1-s)^{\frac{1}{2}}$, and so

$$
\int_{t}^{1}(1-s)^{\frac{1}{2}} d s \leq-\int_{t}^{1} g^{\prime}(s) d s \leq 2 \int_{t}^{1}(1-s)^{\frac{1}{2}} d s
$$

which implies $\frac{2}{3}(1-t)^{\frac{3}{2}} \leq g(t) \leq \frac{4}{3}(1-t)^{\frac{3}{2}}$, since $g(1)=0$.

Note also that, for $z>0$,

$$
\begin{aligned}
& \operatorname{Ai}(-z)=\frac{1}{3} z^{\frac{1}{2}}\left[J_{1 / 3}\left(\frac{2}{3} z^{\frac{3}{2}}\right)+J_{-1 / 3}\left(\frac{2}{3} z^{\frac{3}{2}}\right)\right], \\
& \operatorname{Bi}(-z)=\left(\frac{z}{3}\right)^{\frac{1}{2}}\left[J_{1 / 3}\left(\frac{2}{3} z^{\frac{3}{2}}\right)+J_{-1 / 3}\left(\frac{2}{3} z^{\frac{3}{2}}\right)\right] .
\end{aligned}
$$

3.2.3. Trivial Estimates. It follows from the asymptotics above that for $\kappa$ large we have the following crude estimates for our Laguerre function; see Askey and Wainger [1:

$$
\left|\Lambda_{\kappa}^{\delta}(x)\right| \leq C \begin{cases}(x \nu)^{\frac{\delta}{2}} & \text { if } 0 \leq x \leq \frac{1}{\nu}, \\ (x \nu)^{-\frac{1}{4}} & \text { if } \frac{1}{\nu} \leq x \leq \frac{\nu}{2}, \\ \nu^{-\frac{1}{4}}(\nu-x)^{-\frac{1}{4}} & \text { if } \frac{\nu}{2} \leq x \leq \nu-\nu^{\frac{1}{3}}, \\ \nu^{-\frac{1}{3}} & \text { if } \nu-\nu^{\frac{1}{3}} \leq x \leq \nu+\nu^{\frac{1}{3}}, \\ \nu^{-\frac{1}{4}}(x-\nu)^{-\frac{1}{4}} e^{-\gamma_{1} \nu^{-\frac{1}{2}}(x-\nu)^{\frac{3}{2}}} & \text { if } \nu+\nu^{\frac{1}{3}} \leq x \leq \frac{3 \nu}{2}, \\ e^{-\gamma_{2} x} & \text { if } x \geq \frac{3 \nu}{2},\end{cases}
$$

where $\gamma_{1}, \gamma_{2}>0$ are fixed constants.

\section{Bounded Degree Case: Proof of Theorem 2.1}

We shall now prove Theorem 2.1 and hence assume $\kappa \leq \kappa_{0}$ for some large constant $\kappa_{0}$. To obtain estimates for $\mu(\kappa, \lambda)$ we may, with no loss in generality, assume that $\lambda>0$. If we now perform the rescaling $r \mapsto \lambda^{-\frac{1}{\beta+2}} r$ we see that

$$
\begin{aligned}
\mu\left(\kappa, \lambda^{\beta+2}\right)= & c_{\kappa}^{n-1} \lambda^{\alpha} \int_{0}^{\pi} \int_{0}^{\infty}(\sin \theta)^{n-1} \chi_{\epsilon}\left(\frac{r}{\lambda}\right) r^{-1-\alpha} e^{i \lambda^{\beta} \varphi(r, \theta)}\left(\frac{1}{2} \lambda^{\beta} r^{2} \sin \theta\right)^{\frac{1-n}{2}} \\
& \cdot \Lambda_{\kappa}^{n-1}\left(\frac{1}{2} \lambda^{\beta} r^{2} \sin \theta\right) d r d \theta
\end{aligned}
$$

where $\varphi(r, \theta)=r^{-\beta}+r^{2} \cos \theta$.

It is easy to see that Theorem 2.1 is then equivalent to the following result.

\footnotetext{
4 Recall that $\operatorname{Ai}(z)$ and $\operatorname{Bi}(z)$ are independent solutions of the differential equation $\frac{d^{2} y}{d z^{2}}=z y$ and have the integral representations $\operatorname{Ai}(z)=\frac{1}{\pi} \int_{0}^{\infty} \cos \left(\frac{1}{3} t^{3}+z t\right) d t$ and $\operatorname{Bi}(z)=$ $\frac{1}{\pi} \int_{0}^{\infty}\left\{e^{\frac{1}{3} t^{3}+z t}+\sin \left(\frac{1}{3} t^{3}+z t\right)\right\} d t$.
} 
Theorem 4.1. If $\kappa \leq \kappa_{0}$ and $\lambda \gg 1$, then we have the asymptotic expansion

$$
\mu\left(\kappa, \lambda^{\beta+2}\right)=c_{1} \lambda^{\alpha-\left(n+\frac{1}{2}\right) \beta} e^{i c_{2} \lambda^{\beta}}+O\left(\lambda^{\alpha-(n+1-\varepsilon) \beta}\right) .
$$

Let us introduce some notation. It follows from (4) that for $\lambda>0$

$$
\mu\left(\kappa, \lambda^{\beta+2}\right)=\int_{0}^{\pi}(\sin \theta)^{n-1} \mathcal{I}(\theta) d \theta=\int_{0}^{\infty} \chi_{\epsilon}\left(\frac{r}{\lambda}\right) r^{-1-\alpha} e^{i \lambda^{\beta} r^{-\beta}} \mathcal{J}(r) d r
$$

where

$$
\mathcal{I}=\mathcal{I}(\theta)=\left(c_{\kappa}^{n-1}\right)^{2} \lambda^{\alpha} \int_{0}^{\infty} \chi_{\epsilon}\left(\frac{r}{\lambda}\right) r^{-1-\alpha} e^{i \lambda^{\beta} \varphi(r, \theta)} L_{\kappa}^{n-1}\left(\frac{1}{2} \lambda^{\beta} r^{2} \sin \theta\right) e^{-\frac{1}{4} \lambda^{\beta} r^{2} \sin \theta} d r,
$$

and

$$
\mathcal{J}=\mathcal{J}(r)=\left(c_{\kappa}^{n-1}\right)^{2} \lambda^{\alpha} \int_{0}^{\pi}(\sin \theta)^{n-1} e^{i \lambda^{\beta} r^{2} \cos \theta} L_{\kappa}^{n-1}\left(\frac{1}{2} \lambda^{\beta} r^{2} \sin \theta\right) e^{-\frac{1}{4} \lambda^{\beta} r^{2} \sin \theta} d \theta
$$

Proof of Theorem 4.1, Let $C_{\beta}$ be a suitably small constant to be determined.

Region A: $0 \leq r \leq C_{\beta}$. Provided that $C_{\beta}$ is chosen small enough we have that

$$
-\partial_{r} \varphi(r, \theta)=\beta r^{-(\beta+1)}-2 r \cos \theta \geq C r^{-(\beta+1)} .
$$

It then essentially follows from Proposition 3.1 (as $\chi_{\epsilon}\left(\frac{r}{\lambda}\right)$ and all of its derivatives vanish at $r=0$ ) that

$$
|\mathcal{I}(\theta)| \leq C \lambda^{\alpha-N \beta},
$$

for all $N \geq 0$; we of course then obtain that $\left|\mu\left(\kappa, \lambda^{\beta+2}\right)\right| \leq C \lambda^{\alpha-N \beta}$.

Region B: $C_{\beta} \leq r \leq \lambda$. We now certainly have that $\mu(\kappa, \lambda)$ is absolutely convergent, but now the phase in $\mathcal{I}$ may be critical.

Case 1: If $\lambda^{-\beta(1-\varepsilon)} \leq r^{2} \sin \theta \leq r^{2}$, then $\lambda^{\beta} r^{2} \sin \theta \geq \lambda^{\beta \varepsilon}$. It follows immediately that

$$
L_{\kappa}^{n-1}\left(\frac{1}{2} \lambda^{\beta} r^{2} \sin \theta\right) e^{-\frac{1}{4} \lambda^{\beta} r^{2} \sin \theta} \leq C \lambda^{-N \beta},
$$

for all $N \geq 0$ and hence that $\left|\mu\left(\kappa, \lambda^{\beta+2}\right)\right| \leq C \lambda^{\alpha-N \beta}$.

Case 2: If $0 \leq r^{2} \sin \theta \leq \lambda^{-\beta(1-\varepsilon)}$, then it follows that either $\cos \theta \approx 1$ or $\cos \theta \approx-1$.

(i) We first note that if $\frac{\pi}{2} \leq \theta \leq \pi$, then $\cos \theta \approx-1$, and it follows that

$$
-\partial_{r} \varphi(r, \theta)=\beta r^{-(\beta+1)}-2 r \cos \theta \geq C_{\beta},
$$

and that we can argue as in Region $A$.

(ii) Now if $0 \leq \theta \leq \frac{\pi}{2}$, then $\cos \theta \approx 1$ and we shall write $\mathcal{J}(r)=\mathcal{M}(r)+\mathcal{E}(r)$, where

$$
\begin{gathered}
\mathcal{M}(r)=\left(c_{\kappa}^{n-1}\right)^{2} \lambda^{\alpha} e^{i \lambda^{\beta} r^{2}} \int_{0}^{\frac{\pi}{2}}(\sin \theta)^{n-1} L_{\kappa}^{n-1}\left(\frac{1}{2} \lambda^{\beta} r^{2} \sin \theta\right) e^{-\frac{1}{4} \lambda^{\beta} r^{2} \sin \theta} d \theta \\
\mathcal{E}(r)=\left(c_{\kappa}^{n-1}\right)^{2} \lambda^{\alpha} \int_{0}^{\frac{\pi}{2}}(\sin \theta)^{n-1} L_{\kappa}^{n-1}\left(\frac{1}{2} \lambda^{\beta} r^{2} \sin \theta\right) e^{-\frac{1}{4} \lambda^{\beta} r^{2} \sin \theta}\left[e^{i \lambda^{\beta} r^{2} \cos \theta}-e^{i \lambda^{\beta} r^{2}}\right] d \theta
\end{gathered}
$$

It is then easy to see that

$$
|\mathcal{E}(r)| \leq C \lambda^{\alpha+\beta} r^{2} \int_{0 \leq r^{2} \sin \theta \leq \lambda^{-\beta(1-\varepsilon)}}(\sin \theta)^{n+1} d \theta \leq C \lambda^{\left.\alpha-\left(n+1-\varepsilon^{\prime}\right) \beta\right)} r^{-2 n-2} .
$$


Now for the main term we write $\mathcal{M}(r)=\mathcal{M}_{1}(r)+\mathcal{M}_{2}(r)$, where

$$
\begin{gathered}
\mathcal{M}_{1}(r)=\left(c_{\kappa}^{n-1}\right)^{2} \lambda^{\alpha} e^{i \lambda^{\beta} r^{2}} \int_{0}^{\frac{\pi}{2}}(\sin \theta)^{n-1} L_{\kappa}^{n-1}\left(\frac{1}{2} \lambda^{\beta} r^{2} \sin \theta\right) e^{-\frac{1}{4} \lambda^{\beta} r^{2} \sin \theta} \cos \theta d \theta, \\
\mathcal{M}_{2}(r)=\left(c_{\kappa}^{n-1}\right)^{2} \lambda^{\alpha} \int_{0}^{\frac{\pi}{2}}(\sin \theta)^{n-1} L_{\kappa}^{n-1}\left(\frac{1}{2} \lambda^{\beta} r^{2} \sin \theta\right) e^{-\frac{1}{4} \lambda^{\beta} r^{2} \sin \theta}[1-\cos \theta] d \theta .
\end{gathered}
$$

In a similar manner to the estimate for $\mathcal{E}(r)$ above we obtain the estimate

$$
\left|\mathcal{M}_{2}(r)\right| \leq C \lambda^{\alpha} \int_{0 \leq r^{2} \sin \theta \leq \lambda^{-\beta(1-\varepsilon)}}(\sin \theta)^{n+1} d \theta \leq C \lambda^{\left.\alpha-\left(n+2-\varepsilon^{\prime}\right) \beta\right)} r^{-2 n-4} .
$$

Now for the main term $\mathcal{M}_{1}(r)$ we have

$$
\begin{aligned}
\mathcal{M}_{1}(r) & =\left(c_{\kappa}^{n-1}\right)^{2} \lambda^{\alpha} e^{i \lambda^{\beta} r^{2}} \int_{0 \leq r^{2} \sin \theta \leq \lambda^{-\beta(1-\varepsilon)}}(\sin \theta)^{n-1} L_{\kappa}^{n-1}\left(\frac{1}{2} \lambda^{\beta} r^{2} \sin \theta\right) e^{-\frac{1}{4} \lambda^{\beta} r^{2} \sin \theta} \cos \theta d \theta \\
& =\left(c_{\kappa}^{n-1}\right)^{2} \lambda^{\alpha} e^{i \lambda^{\beta} r^{2}} \lambda^{-n \beta} r^{-2 n} \int_{0}^{\lambda^{\beta \varepsilon}} u^{n-1} L_{\kappa}^{n-1}\left(\frac{1}{2} u\right) e^{-\frac{1}{4} u} d u \\
& =C \lambda^{\alpha-n \beta} e^{i \lambda^{\beta} r^{2}} r^{-2 n}+O\left(\lambda^{\alpha-N \beta}\right) .
\end{aligned}
$$

We have therefore shown that

$$
\mu\left(\kappa, \lambda^{\beta+2}\right)=C \lambda^{\alpha-n \beta} M+O\left(\lambda^{\alpha-(n+1-\varepsilon) \beta)}\right),
$$

where

$$
M=\int_{0}^{\infty} \chi_{\epsilon}\left(\frac{r}{\lambda}\right) r^{-2 n-1-\alpha} e^{i \lambda^{\beta} \varphi(r, 0)} d r
$$

Now notice that

$$
\partial_{r}^{2} \varphi(r, 0)=\beta(\beta+1) r^{-(\beta+2)}+2 \geq 2 ;
$$

it follows that $\partial_{r} \varphi(r, 0)$ can vanish at most one point $r=r_{0}$. Suppose that we are in a suitably small neighborhood of $r_{0}$; outside of this interval we have that $\left|\partial_{r} \varphi(r, 0)\right| \geq C>0$ and can argue as in Region A. Note that for all $\ell=0,1, \ldots$

$$
\left|\partial_{r}^{\ell} \varphi(r, 0)\right|+\left|\partial_{r}^{\ell}\left[\chi_{\epsilon}\left(\frac{r}{\lambda}\right) r^{-2 n-1-\alpha}\right]\right| \leq c_{\ell} .
$$

We may therefore, in a suitably small neighborhood of $r_{0}$, apply the method of stationary phase (Proposition 3.3) and obtain that

$$
M=C\left(r_{0}\right) \lambda^{-\frac{1}{2} \beta} e^{i \lambda^{\beta} \varphi\left(r_{0}, 0\right)}+O\left(\lambda^{-\frac{3}{2} \beta}\right) .
$$

\section{Unbounded degree case: Proof of Theorem 2.2}

We shall now prove Theorem 2.2, and hence assume $\kappa \geq \kappa_{0}$, for some large constant $\kappa_{0}$. We may, as in Section [4, assume $\lambda>0$. Performing the rescaling $r \mapsto 2^{-j} r$ we see that

$$
\mu_{j}\left(\kappa, \lambda 2^{2 j}\right)=c_{\kappa}^{n-1} 2^{j \alpha} \int_{0}^{\pi} \int_{0}^{\infty}(\sin \theta)^{n-1} \vartheta(r) r^{-1-\alpha} e^{i 2^{j \beta} \varphi(r, \theta)} x^{\frac{1-n}{2}} \Lambda_{\kappa}^{n-1}(x) d r d \theta,
$$

where now

$$
\varphi(r, \theta)=r^{-\beta}+\lambda 2^{-j \beta} r^{2} \cos \theta \quad \text { and } \quad x=x(\lambda, r, \theta)=\frac{1}{2} \lambda r^{2} \sin \theta .
$$

It is then easy to see that Theorem 2.2 will be a consequence of the following result. 
Theorem 5.1. If $\kappa \geq \kappa_{0}$, then the following estimate holds uniformly in $\kappa$ and $\lambda \neq 0$ :

$$
\left|\mu_{j}\left(\kappa, \lambda 2^{2 j}\right)\right| \leq C 2^{j \alpha} \min \left\{2^{-j n \beta},(1+\lambda \kappa)^{-\frac{n}{2}}\right\} .
$$

In order to estimate $\mu_{j}\left(\kappa, \lambda 2^{2 j}\right)$ we shall make use of the asymptotics for Laguerre functions presented in $\$ 3.2$. It is then natural to consider six separate regions and write

where for $k=1, \ldots, 6$,

$$
\mu_{j}\left(\kappa, \lambda 2^{2 j}\right)=\mu_{j, 1}\left(\kappa, \lambda 2^{2 j}\right)+\cdots+\mu_{j, 6}\left(\kappa, \lambda 2^{2 j}\right)
$$

$$
\begin{aligned}
\mu_{j, k}\left(\kappa, \lambda 2^{2 j}\right)= & c_{\kappa}^{n-1} 2^{j \alpha} \int_{0}^{\pi} \int_{0}^{\infty} \eta_{k}(x)(\sin \theta)^{n-1} \vartheta(r) r^{-1-\alpha} \\
& \cdot e^{i 2^{j \beta} \varphi(r, \theta)} x^{\frac{1-n}{2}} \Lambda_{\kappa}^{n-1}(x) d r d \theta,
\end{aligned}
$$

and each $\eta_{k}(x)$ smoothly localizes $x$ to the $k$ th interval indicated in 3.2 .3 .

As in Section 4 we write, for $\lambda>0$,

$$
\mu_{j, k}\left(\kappa, \lambda 2^{2 j}\right)=\int_{0}^{\pi}(\sin \theta)^{n-1} \mathcal{I}_{k}(j, \theta) d \theta=\int_{0}^{\infty} \vartheta(r) r^{-1-\alpha} e^{i 2^{j \beta} r^{-\beta}} \mathcal{J}_{k}(j, r) d r,
$$

where, as we are now considering the case when $\kappa \geq \kappa_{0}$, we essentially have

$$
\begin{gathered}
\mathcal{I}_{k}=\mathcal{I}_{k}(j, \theta)=2^{j \alpha} \int_{0}^{\infty} \eta_{k}(x) \vartheta(r) r^{-1-\alpha} e^{i 2^{j \beta} \varphi(r, \theta)}(x \nu)^{\frac{1-n}{2}} \Lambda_{\kappa}^{n-1}(x) d r, \\
\mathcal{J}_{k}=\mathcal{J}_{k}(j, r)=2^{j \alpha} \int_{0}^{\pi} \eta_{k}(x)(\sin \theta)^{n-1} e^{i \lambda r^{2} \cos \theta}(x \nu)^{\frac{1-n}{2}} \Lambda_{\kappa}^{n-1}(x) d \theta .
\end{gathered}
$$

It will again be natural to consider separately the regions where we can and cannot integrate by parts in $r$. It is clear that we may choose a constant $C_{\beta}$ small enough so that

$$
-\partial_{r} \varphi(r, \theta) \geq C r^{-(\beta+1)} \quad \text { and } \quad\left|\partial_{r}^{\ell} \varphi(r, \theta)\right| \leq C r^{-(\beta+\ell)},
$$

whenever $0<\lambda 2^{-j \beta} \leq C_{\beta}$. However if we are to integrate by parts in $r$ we must take care of what happens when the derivative hits the amplitude of $\mathcal{I}_{k}$.

Recall that $\Lambda_{\kappa}^{\delta}(x)=c_{\kappa}^{\delta} L_{\kappa}^{\delta}(x) e^{-\frac{1}{2} x} x^{\frac{\delta}{2}}$. Now since $\frac{d}{d x} L_{\kappa}^{\delta}(x)=-L_{\kappa-1}^{\delta+1}(x)$ and $c_{\kappa-1}^{\delta+1}=\kappa^{-\frac{1}{2}} c_{\kappa}^{\delta}$ it follows that

$$
\frac{d}{d x} \Lambda_{\kappa}^{\delta}(x)=\frac{1}{2}\left(\frac{\delta}{x}-1\right) \Lambda_{\kappa}^{\delta}(x)-\left(\frac{\kappa}{x}\right)^{\frac{1}{2}} \Lambda_{\kappa-1}^{\delta+1}(x) .
$$

Therefore, using the fact that $\partial_{r} x=\frac{2 x}{r}$ we see that

$$
\partial_{r} \Lambda_{\kappa}^{\delta}(x)=-r^{-1}\left[(x-\delta) \Lambda_{\kappa}^{\delta}(x)+2(x \kappa)^{\frac{1}{2}} \Lambda_{\kappa-1}^{\delta+1}(x)\right] .
$$

If we instead take $N$ derivatives it is easy to see that we get

$\partial_{r}^{N} \Lambda_{\kappa}^{\delta}(x)=r^{-N}\left[P_{N}(x) \Lambda_{\kappa}^{\delta}(x)+(x \kappa)^{\frac{1}{2}} P_{N-1}(x) \Lambda_{\kappa-1}^{\delta+1}(x)+\cdots+(x \kappa)^{\frac{N}{2}} P_{0}(x) \Lambda_{\kappa-N}^{\delta+N}(x)\right]$, that is,

$$
\partial_{r}^{N} \Lambda_{\kappa}^{\delta}(x)=r^{-N} \sum_{\ell=0}^{N}(x \kappa)^{\frac{\ell}{2}} P_{N-\ell}(x) \Lambda_{\kappa-\ell}^{\delta+\ell}(x),
$$

where $P_{N-\ell}(x)$ is some polynomial of degree $N-\ell$ in $x$. It is therefore only advantageous to integration by parts in $r$ when both $x$ and $(x \nu)^{\frac{1}{2}}$ are dominated by $2^{j \beta}$ and hence we shall in general only do so when

$$
\max \left\{(\lambda \nu)^{\frac{1}{2}}, \lambda\right\} \leq C_{\beta} 2^{j \beta} .
$$


In actual fact we shall establish, for each $k$, the following:

$$
\left|\mu_{j, k}\left(\kappa, \lambda 2^{2 j}\right)\right| \leq C 2^{j \alpha} \begin{cases}2^{-j n \beta} & \text { if } \max \left\{(\lambda \nu)^{\frac{1}{2}}, \lambda\right\} \leq C_{\beta} 2^{j \beta}, \\ \min \left\{(\lambda \nu)^{-\frac{n}{2}}, \lambda^{-n}\right\} & \text { if } \max \left\{(\lambda \nu)^{\frac{1}{2}}, \lambda\right\} \geq C_{\beta} 2^{j \beta},\end{cases}
$$

and from this one easily establishes Theorem 5.1 .

5.1. Neighborhood of $0: 0 \leq x \leq \frac{1}{\nu}$. Notice that here $x \nu \leq 1$ and $\left|\Lambda_{\kappa}^{\delta}(x)\right| \leq$ $C(x \nu)^{\frac{\delta}{2}}$.

Region $A: \max \left\{(\lambda \nu)^{\frac{1}{2}}, \lambda\right\} \leq C_{\beta} 2^{j \beta}$. In this region we can integrate by parts. Using our trivial estimate it is easy to see that

$$
\left|\partial_{r}^{N} \Lambda_{\kappa}^{\delta}(x)\right| \leq C(x \nu)^{\frac{\delta}{2}+N} .
$$

Therefore integrating by parts $N$ times we obtain the estimate

$$
\begin{aligned}
\int_{0}^{\pi}(\sin \theta)^{n-1}\left|\mathcal{I}_{1}(\theta)\right| d \theta & =2^{j \alpha} \int_{0}^{\pi}(\sin \theta)^{n-1} \\
& \cdot\left|\int_{0}^{\infty} \eta_{1}(x) \vartheta(r) r^{-1-\alpha} e^{i 2^{j \beta} \varphi(r, \theta)}(x \nu)^{\frac{1-n}{2}} \Lambda_{\kappa}^{n-1}(x) d r\right| d \theta \\
\leq & C 2^{j(\alpha-N \beta)} \int_{\sin \theta \leq \min \left\{1,(\lambda \nu)^{-1}\right\}}(\sin \theta)^{n-1} d \theta \\
\leq & C 2^{j(\alpha-N \beta)}(1+\lambda \nu)^{-n},
\end{aligned}
$$

for any $N \geq 0$. So we obtain the estimate

$$
\left|\mu_{j, 1}\left(\kappa, \lambda 2^{2 j}\right)\right| \leq C 2^{j(\alpha-N \beta)}(1+\lambda \nu)^{-n} .
$$

Region $B: \max \left\{(\lambda \nu)^{\frac{1}{2}}, \lambda\right\} \geq C_{\beta} 2^{j \beta}$. Here we necessarily have $\sin \theta \leq 2(\lambda \nu)^{-1} \leq$ $2^{-j \beta}$ and therefore either $\cos \theta \approx 1$ or $\cos \theta \approx-1$.

(i) We first note that if $\frac{\pi}{2} \leq \theta \leq \pi$, then $\cos \theta \approx-1$, and it follows that

$$
-\partial_{r} \varphi(r, \theta)=\beta r^{-(\beta+1)}-2 \lambda 2^{-j \beta} r \cos \theta \geq C\left(1+\lambda 2^{-j \beta}\right) .
$$

We can therefore argue as in Region $A$ and obtain the estimate

$$
\left|\mu_{j, 1}\left(\kappa, \lambda 2^{2 j}\right)\right| \leq C 2^{j \alpha}(\lambda \nu)^{-n} \min \left\{2^{-j N \beta}, \lambda^{-N}\right\} .
$$

(ii) Now if $0 \leq \theta \leq \frac{\pi}{2}$, then $\cos \theta \approx 1$ and it is easy to see that

$$
\partial_{r}^{2} \varphi(r, \theta)=\beta r^{-(\beta+1)}+2 \lambda 2^{-j \beta} \cos \theta \geq C\left(1+\lambda 2^{-j \beta}\right),
$$

and hence by van der Corput's lemma we have $\left|\mathcal{I}_{1}(\theta)\right| \leq C 2^{j \alpha} \min \left\{2^{-j \frac{1}{2} \beta}, \lambda^{-\frac{1}{2}}\right\}$. It therefore follows that

$$
\begin{aligned}
\left|\mu_{j, 1}\left(\kappa, \lambda 2^{2 j}\right)\right| & \leq C \int_{0}^{\pi}(\sin \theta)^{n-1}\left|\mathcal{I}_{1}(\theta)\right| d \theta \\
& \leq C 2^{j \alpha} \min \left\{2^{-j \frac{1}{2} \beta}, \lambda^{-\frac{1}{2}}\right\} \int_{\sin \theta \leq(\lambda \nu)^{-1}}(\sin \theta)^{n-1} d \theta \\
& \leq C 2^{j \alpha}(\lambda \nu)^{-n} \min \left\{2^{-j \frac{1}{2} \beta}, \lambda^{-\frac{1}{2}}\right\} .
\end{aligned}
$$


5.2. Oscillatory interval I: $\frac{1}{\nu} \leq x \leq \frac{\nu}{2}$. Notice that here $\frac{\nu}{2} \leq \nu-x<\nu$, and $\left|\Lambda_{\kappa}^{\delta}(x)\right| \leq C(x \nu)^{-\frac{1}{4}}$.

In this interval we shall make explicit use of the oscillation in the main term of our asymptotic expansion, which in this case is given in terms of Bessel functions; see $\$ 3.2 .1$. We note here that from (6) and Lemma 3.5 it follows that

$$
\psi^{\prime} \sim\left(\frac{\nu}{x}\right)^{\frac{1}{2}} \text { while } \quad \psi \sim\left(\frac{x}{\nu}\right)^{\frac{1}{2}} .
$$

The following estimates are then immediate:

$$
\begin{gathered}
(\nu \psi)^{-\frac{1}{2}} \sim(x \nu)^{-\frac{1}{4}} \quad \text { and } \quad \partial_{r}\left[(\nu \psi)^{-\frac{1}{2}}\right]=-\frac{x}{r \nu^{\frac{3}{2}}} \psi^{-\frac{3}{2}} \psi^{\prime} \sim-(x \nu)^{-\frac{1}{4}}, \\
\partial_{r} \nu \psi=\frac{1}{r} x^{\frac{1}{2}}(\nu-x)^{\frac{1}{2}} \sim(x \nu)^{\frac{1}{2}} \quad \text { and } \quad \partial_{r}^{2} \nu \psi=-\frac{1}{r^{2}} \frac{x^{\frac{3}{2}}}{(\nu-x)^{\frac{1}{2}}} \sim x^{\frac{3}{2}} \nu^{-\frac{1}{2}}, \\
\left(\frac{\nu}{x}\right)^{\frac{1}{2}}\left(\frac{\psi}{\psi^{\prime}}\right)^{\frac{1}{2}} \leq C \quad \text { and } \quad\left|\partial_{r}\left[\left(\frac{\nu}{x}\right)^{\frac{1}{2}}\left(\frac{\psi}{\psi^{\prime}}\right)^{\frac{1}{2}}\right]\right| \leq C .
\end{gathered}
$$

Region $A: \max \left\{(\lambda \nu)^{\frac{1}{2}}, \lambda\right\} \leq C_{\beta} 2^{j \beta}$. In this region we shall again integrate by parts in $r$ and since we have $x^{N} \leq(x \nu)^{\frac{N}{2}}$ it suffices to consider the integral

$$
I_{2}(\theta)=2^{j(\alpha-N \beta)} \int_{0}^{\infty} \eta_{2}(x) \vartheta(r) e^{i 2^{j \beta} \varphi(r, \theta)}(x \nu)^{\frac{N+1-n}{2}} \Lambda_{\kappa-N}^{n-1+N}(x) d r .
$$

Using the Bessel asymptotic forms (5) we may write $I_{2}=c B+E_{B}$, where

$$
B(\theta)=2^{j(\alpha-N \beta)} \int_{0}^{\infty} \eta_{2}(x) \vartheta(r) e^{i 2^{j \beta} \varphi(r, \theta)}(x \nu)^{\frac{N+1-n}{2}}\left(\frac{\nu}{x}\right)^{\frac{1}{2}}\left(\frac{\psi}{\psi^{\prime}}\right)^{\frac{1}{2}} J_{n-1+N}(\nu \psi) d r .
$$

ERROR TERM:

$$
\begin{aligned}
\left|E_{B}(\theta)\right| \leq & C 2^{j(\alpha-N \beta)} \int_{0}^{\infty} \eta_{2}(x) \vartheta(r)(x \nu)^{\frac{N+1-n}{2}}\left(\frac{\nu}{x}\right)^{\frac{1}{2}}\left(\frac{\psi}{\psi^{\prime}}\right)^{\frac{1}{2}} \\
& \cdot \nu^{-1}\left(\frac{x}{\nu-x}\right)^{\frac{1}{2}}\left|\widetilde{J}_{n-1+N}(\nu \psi)\right| d r \\
\leq & C 2^{j(\alpha-N \beta)} \nu^{-1} \int_{x \leq \nu}(x \nu)^{\frac{1}{2}\left(N-n-\frac{1}{2}\right)} d x \\
\leq & C 2^{j\left(\alpha-\left(n+\frac{1}{2}\right) \beta\right)}
\end{aligned}
$$

provided $N>n$, since $(x \nu)^{\frac{1}{2}} \leq C 2^{j \beta}$.

MAIN TERM: Since $\nu \psi \geq(x \nu)^{\frac{1}{2}} \geq 1$ we shall utilize expression (3) and write $B=B_{1}+B_{2}$, where

$$
\begin{aligned}
B_{1}(\theta)= & 2^{j(\alpha-N \beta)} \int_{0}^{\infty} \eta_{2}(x) \vartheta(r) \\
& \cdot e^{i 2^{j \beta}\left[\varphi(r, \theta)+2^{-j \beta} \nu \psi\right]}(x \nu)^{\frac{N+1-n}{2}}\left(\frac{\nu}{x}\right)^{\frac{1}{2}}\left(\frac{\psi}{\psi^{\prime}}\right)^{\frac{1}{2}} \sigma_{1}(\nu \psi) d r \\
B_{2}(\theta)= & 2^{j(\alpha-N \beta)} \int_{0}^{\infty} \eta_{2}(x) \vartheta(r) \\
& \cdot e^{i 2^{j \beta}\left[\varphi(r, \theta)-2^{-j \beta} \nu \psi\right]}(x \nu)^{\frac{N+1-n}{2}}\left(\frac{\nu}{x}\right)^{\frac{1}{2}}\left(\frac{\psi}{\psi^{\prime}}\right)^{\frac{1}{2}} \sigma_{2}(\nu \psi) d r,
\end{aligned}
$$


where each $\sigma_{i}$ is a symbol of order $-\frac{1}{2}$. Since $\partial_{r} \psi \sim \frac{1}{r} \psi$ it follows that

$$
\left|\partial_{r}^{\ell} \sigma_{i}(\nu \psi)\right| \leq C r^{-\ell}(\nu \psi)^{-\frac{1}{2}} .
$$

Let us first consider the integral $B_{1}$, and let $\Phi_{\theta}(r)=\varphi(r, \theta)+2^{-j \beta} \nu \psi$. Now

$$
\partial_{r} \Phi_{\theta}(r)=-\beta r^{-(\beta+1)}+\lambda 2^{-j \beta} 2 r \cos \theta+2^{-j \beta} \frac{1}{r} x^{\frac{1}{2}}(\nu-x)^{\frac{1}{2}},
$$

and so if $C_{\beta}$ is chosen small enough it follows that

$$
\left|\partial_{r} \Phi_{\theta}(r)\right| \geq C>0
$$

In addition to this we also have

$$
\partial_{r}^{2} \Phi_{\theta}(r)=\beta(\beta+1) r^{-(\beta+2)}+\lambda 2^{-j \beta} 2 \cos \theta-2^{-j \beta} \frac{1}{r^{2}} \frac{x^{\frac{3}{2}}}{(\nu-x)^{\frac{1}{2}}} \geq C>0 .
$$

If we let

$$
\Psi_{\theta}(r)=\vartheta(r)(x \nu)^{\frac{N+1-n}{2}}\left(\frac{\nu}{x}\right)^{\frac{1}{2}}\left(\frac{\psi}{\psi^{\prime}}\right)^{\frac{1}{2}} \sigma_{1}(\nu \psi),
$$

then for all $\ell=0,1, \ldots$

$$
\left|\partial_{r}^{\ell} \Psi_{\theta}(r)\right| \leq C(x \nu)^{\frac{N}{2}}(\nu \psi)^{-\frac{1}{2}} .
$$

Applying van der Corput's lemma (integration by parts) gives

$$
\left|B_{1}(\theta)\right| \leq C 2^{j(\alpha-(N+1) \beta)} \int_{0}^{\infty} \vartheta(r)(x \nu)^{\frac{2 N+1-2 n}{4}} d r \leq C 2^{j\left(\alpha-\left(n+\frac{1}{2}\right) \beta\right)},
$$

again provided $N>n$. Of course the phase in $B_{2}$ is never stationary, so we obtain the same estimate for $B_{2}$; in Region $A$ we therefore obtain

$$
\left|\mu_{j, 2}\left(\kappa, \lambda 2^{2 j}\right)\right| \leq C 2^{j\left(\alpha-\left(n+\frac{1}{2}\right) \beta\right)} .
$$

Region $B: \max \left\{(\lambda \nu)^{\frac{1}{2}}, \lambda\right\} \geq C_{\beta} 2^{j \beta}$. Here we shall not integrate by parts first and so we wish to estimate

$$
\mathcal{J}_{2}=2^{j \alpha} \int_{0}^{\pi} \eta_{2}(x)(\sin \theta)^{n-1} e^{i \lambda r^{2} \cos \theta}(x \nu)^{\frac{1-n}{2}} \Lambda_{\kappa}^{n-1}(x) d \theta .
$$

Using the asymptotic forms for our Laguerre functions we can write $\mathcal{J}_{2}=c \mathcal{B}+\mathcal{E}_{B}$, where

$$
\mathcal{B}(r)=2^{j \alpha} \int_{0}^{\pi} \eta_{2}(x)(\sin \theta)^{n-1} e^{i \lambda r^{2} \cos \theta}(x \nu)^{\frac{1-n}{2}}\left(\frac{\nu}{x}\right)^{\frac{1}{2}}\left(\frac{\psi}{\psi^{\prime}}\right)^{\frac{1}{2}} J_{n-1}(\nu \psi) d \theta .
$$

ERROR TERM:

$$
\begin{aligned}
\left|\mathcal{E}_{B}(r)\right| & \leq C 2^{j \alpha} \int_{0}^{\pi} \eta_{2}(x)(\sin \theta)^{n-1}(x \nu)^{\frac{1-n}{2}}\left(\frac{\nu}{x}\right)^{\frac{1}{2}}\left(\frac{\psi}{\psi^{\prime}}\right)^{\frac{1}{2}} \nu^{-1}\left(\frac{x}{\nu-x}\right)^{\frac{1}{2}}\left|\widetilde{J}_{n-1}(\nu \psi)\right| d \theta \\
& \leq C 2^{j \alpha} \int_{\sin \theta \leq \min \left\{1, \nu \lambda^{-1}\right\}}(\sin \theta)^{n-1}(x \nu)^{-\frac{1}{2}\left(n+\frac{1}{2}\right)} d \theta \\
& \leq C 2^{j \alpha} \min \left\{(\lambda \nu)^{-\frac{1}{2}\left(n+\frac{1}{2}\right)}, \lambda^{-n} \nu^{-\frac{1}{2}}\right\} .
\end{aligned}
$$

MAIN TERM : Since $\nu \psi \geq(x \nu)^{\frac{1}{2}} \geq 1$ we shall, as before, write $\mathcal{B}=\mathcal{B}_{1}+\mathcal{B}_{2}$, where

$$
\begin{aligned}
& \mathcal{B}_{1}(r)=2^{j \alpha} \int_{0}^{\pi} \eta_{2}(x)(\sin \theta)^{n-1} e^{i\left[\lambda r^{2} \cos \theta+\nu \psi\right]}(x \nu)^{\frac{1-n}{2}}\left(\frac{\nu}{x}\right)^{\frac{1}{2}}\left(\frac{\psi}{\psi^{\prime}}\right)^{\frac{1}{2}} \sigma_{1}(\nu \psi) d \theta, \\
& \mathcal{B}_{2}(r)=2^{j \alpha} \int_{0}^{\pi} \eta_{2}(x)(\sin \theta)^{n-1} e^{i\left[\lambda r^{2} \cos \theta-\nu \psi\right]}(x \nu)^{\frac{1-n}{2}}\left(\frac{\nu}{x}\right)^{\frac{1}{2}}\left(\frac{\psi}{\psi^{\prime}}\right)^{\frac{1}{2}} \sigma_{2}(\nu \psi) d \theta .
\end{aligned}
$$


Let us first consider the integral $\mathcal{B}_{1}$. If we let $\Phi_{r}(\theta)=\lambda r^{2} \cos \theta+\nu \psi$, then we have

$$
\begin{aligned}
\partial_{\theta} \Phi_{r}(\theta) & =-\lambda r^{2} \sin \theta+\nu\left(\frac{\nu-x}{x}\right)^{\frac{1}{2}} \frac{\lambda r^{2} \cos \theta}{2 \nu} \\
& =-\frac{1}{2} \lambda r^{2}\left(2 \sin \theta-\left(\frac{\nu-x}{x}\right)^{\frac{1}{2}} \cos \theta\right) .
\end{aligned}
$$

We shall now write $\mathcal{B}_{1}=\mathcal{B}_{1}^{1}+\mathcal{B}_{1}^{2}$, where

$$
\begin{gathered}
\mathcal{B}_{1}^{1}(r)=2^{j \alpha} \int_{0}^{\pi} \chi(\sin \theta) \Psi_{r}(\theta) e^{i \Phi_{r}(\theta)} d \theta, \\
\mathcal{B}_{1}^{2}(r)=2^{j \alpha} \int_{0}^{\pi}(1-\chi(\sin \theta)) \Psi_{r}(\theta) e^{i \Phi_{r}(\theta)} d \theta,
\end{gathered}
$$

and

$$
\Psi_{r}(\theta)=(\sin \theta)^{n-1}(x \nu)^{\frac{1-n}{2}}\left(\frac{\nu}{x}\right)^{\frac{1}{2}}\left(\frac{\psi}{\psi^{\prime}}\right)^{\frac{1}{2}} \sigma_{1}(\nu \psi) .
$$

Case 1: If $0 \leq \sin \theta \leq \frac{1}{10}$, then there are two possibilities; $\cos \theta \approx 1$ and $\cos \theta \approx-1$.

(i) If $0 \leq \theta \leq \frac{\pi}{2}$, then $\cos \theta \approx 1$ and

$$
\left(\frac{\nu-x}{x}\right)^{\frac{1}{2}} \cos \theta \geq \frac{1}{2}
$$

it therefore follows that

$$
\left|\partial_{\theta} \Phi_{r}(\theta)\right| \geq(\sin \theta)^{-1}(x \nu)^{\frac{1}{2}}
$$

(ii) If $\frac{\pi}{2} \leq \theta \leq \pi$, then $\cos \theta \approx-1$, and we trivially have the same estimate as above.

Notice also that

$$
\left|\partial_{\theta}^{2} \Phi_{r}(\theta)\right| \leq C(\sin \theta)^{-2}(x \nu)^{\frac{1}{2}}
$$

and since for all $\ell=0,1, \ldots$ we have that $\left|\partial_{\theta}^{\ell} \sigma_{i}(\nu \psi)\right| \leq c_{\ell}(\sin \theta)^{-\ell}(x \nu)^{-\frac{1}{4}}$ it follows that if we let $\Psi_{r}^{1}(\theta)=\chi(\sin \theta) \Psi_{r}(\theta)$, then we have

$$
\begin{gathered}
\left|\Psi_{r}^{1}(\theta)\right| \leq(\sin \theta)^{n-1}(x \nu)^{\frac{1-n}{2}-\frac{1}{4}} \\
\left|\partial_{\theta} \Psi_{r}^{1}(\theta)\right| \leq(\sin \theta)^{n-2}(x \nu)^{\frac{1-n}{2}-\frac{1}{4}}
\end{gathered}
$$

An application of integration by parts therefore gives

$$
\begin{aligned}
\left|\mathcal{B}_{1}^{1}(r)\right| & \leq C 2^{j \alpha} \int_{0}^{\pi} \eta_{2}(x)\left|\partial_{\theta}\left(\left[\partial_{\theta} \Phi_{r}(\theta)\right]^{-1} \Psi_{r}^{1}(\theta)\right)\right| d \theta \\
& \leq C 2^{j \alpha} \int_{\sin \theta \leq \min \left\{1, \nu \lambda^{-1}\right\}}(\sin \theta)^{n-1}(x \nu)^{\frac{1-n}{2}-\frac{3}{4}} d \theta \\
& \leq C 2^{j \alpha} \min \left\{(\lambda \nu)^{-\frac{1}{2}\left(n+\frac{1}{2}\right)}, \lambda^{-n} \nu^{-\frac{1}{2}}\right\} .
\end{aligned}
$$

Case 2: If $\frac{1}{10} \leq \sin \theta \leq 1$, then $\lambda \leq 10 \nu$ and $\Phi_{r}(\theta)$ may now be stationary. However we do have that

$$
\left|\partial_{\theta}^{2} \Phi_{r}(\theta)\right| \geq C(x \nu)^{\frac{1}{2}} \geq C(\lambda \nu)^{\frac{1}{2}}
$$

and if we now let $\Psi_{r}^{2}(\theta)=\chi(\sin \theta) \Psi_{r}(\theta)$ it follows that

$$
\left|\Psi_{r}^{2}(\theta)\right|+\left|\partial_{\theta} \Psi_{r}^{2}(\theta)\right| \leq C(x \nu)^{-\frac{1}{2}\left(n-\frac{1}{2}\right)} \leq(\lambda \nu)^{-\frac{1}{2}\left(n-\frac{1}{2}\right)} .
$$


It therefore follows from an application of van der Corput's lemma that

$$
\left|\mathcal{B}_{1}^{2}(r)\right| \leq C 2^{j \alpha}(\lambda \nu)^{-\frac{1}{4}}(\lambda \nu)^{-\frac{1}{2}\left(n-\frac{1}{2}\right)} \leq C(\lambda \nu)^{-\frac{n}{2}} .
$$

Bringing these two cases together we see that

$$
\left|\mathcal{B}_{1}(r)\right| \leq C 2^{j \alpha} \min \left\{(\lambda \nu)^{-\frac{n}{2}}, \lambda^{-n} \nu^{-\frac{1}{2}}\right\} .
$$

The integral $\mathcal{B}_{2}$ can be treated in exactly the same manner as above. In Region $B$ we therefore obtain

$$
\left|\mu_{j, 2}\left(\kappa, \lambda 2^{2 j}\right)\right| \leq C 2^{j \alpha} \min \left\{(\lambda \nu)^{-\frac{n}{2}}, \lambda^{-n} \nu^{-\frac{1}{2}}\right\} .
$$

5.3. Oscillatory interval II: $\frac{\nu}{2} \leq x \leq \nu-\nu^{\frac{1}{3}}$. We now have $\nu^{\frac{1}{3}} \leq \nu-x \leq \frac{\nu}{2}$ and $\left|\Lambda_{\kappa}^{\delta}(x)\right| \leq C x^{-\frac{1}{4}}(\nu-x)^{-\frac{1}{4}}$. Notice also that $(\lambda \nu)^{\frac{1}{2}} \leq 2 \lambda$.

The situation here is much the same as it was in $\$ 5.2$ only here we must use instead the Airy asymptotic form. In order to do better than the trivial estimate we shall again make use of the oscillation in the main term of our asymptotic expansion. It follows from Lemma 3.6 that

$$
\phi \sim \frac{\nu-x}{\nu}
$$

from this and (8) it is immediately clear that

$$
\frac{1}{10} \leq \phi^{\prime} \leq 10 \quad \text { and } \quad \phi^{\prime \prime} \leq C .
$$

Region $A: \lambda \leq C_{\beta} 2^{j \beta}$. In this region we should integrate by parts and since $x \leq$ $(x \nu)^{\frac{1}{2}}$ it suffices to estimate

$$
I_{3}(\theta)=2^{j(\alpha-N \beta)} \int_{0}^{\infty} \eta_{3}(x) \vartheta(r) e^{i 2^{j \beta} \varphi(r, \theta)}(x \nu)^{\frac{N+1-n}{2}} \Lambda_{\kappa-N}^{n-1+N}(x) d r .
$$

Using the Airy asymptotic forms (7) we may write this as $I_{3}=c A+E_{A}$, where

$$
A(\theta)=2^{j(\alpha-N \beta)} \int_{0}^{\infty} \eta_{3}(x) \vartheta(r) e^{i 2^{j \beta} \varphi(r, \theta)}(x \nu)^{\frac{N+1-n}{2}} x^{-\frac{1}{2}} \nu^{\frac{1}{6}}\left(\frac{1}{-\phi^{\prime}}\right)^{\frac{1}{2}} \operatorname{Ai}\left(-\nu^{\frac{2}{3}} \phi\right) d r .
$$

ERROR TERM:

$$
\begin{aligned}
\left|E_{A}(\theta)\right| & \leq C 2^{j(\alpha-N \beta)} \int_{0}^{\infty} \eta_{3}(x) \vartheta(r)(x \nu)^{\frac{N+1-n}{2}} x^{-\frac{3}{2}} \nu^{\frac{1}{6}}\left(\frac{1}{-\phi^{\prime}}\right)^{\frac{1}{2}}\left|\widetilde{\mathrm{Ai}}\left(-\nu^{\frac{2}{3}} \phi\right)\right| d r \\
& \leq C 2^{j(\alpha-N \beta)} \int_{0}^{\infty} \eta_{3}(x) \vartheta(r)(x \nu)^{\frac{1}{2}\left(N-\frac{1}{3}-n\right)}\left(\nu^{\frac{2}{3}} \phi\right)^{\frac{1}{2}}\left(\nu \phi^{\frac{3}{2}}\right)^{-\frac{1}{2}} d r \\
& \leq C 2^{j(\alpha-N \beta)} \int_{0}^{\infty} \eta_{3}(x) \vartheta(r)(x \nu)^{\frac{1}{2}\left(N-\frac{1}{2}-n\right)} \nu^{\frac{1}{4}}(\nu-x)^{-\frac{1}{4}} d r \\
& \leq C 2^{j\left(\alpha-\left(n+\frac{1}{2}\right) \beta\right)} \nu^{-\frac{3}{4}} \int_{\nu-x \leq \nu}(\nu-x)^{-\frac{1}{4}} d x \\
& \leq C 2^{j\left(\alpha-\left(n+\frac{1}{2}\right) \beta\right)}
\end{aligned}
$$

provided $N>n$.

MAIN TERM: Recall that for $z>0$

$$
\operatorname{Ai}(-z)=\frac{1}{3} z^{\frac{1}{2}}\left[J_{1 / 3}\left(\frac{2}{3} z^{\frac{3}{2}}\right)+J_{-1 / 3}\left(\frac{2}{3} z^{\frac{3}{2}}\right)\right] .
$$


Since $J_{1 / 3}$ and $J_{-1 / 3}$ satisfy the same bounds for large $z$ it suffices to estimate

$$
\begin{aligned}
\widetilde{A}(\theta)= & 2^{j(\alpha-N \beta)} \int_{0}^{\infty} \eta_{3}(x) \vartheta(r) e^{i 2^{j \beta} \varphi(r, \theta)}(x \nu)^{\frac{N+1-n}{2}} \\
& \cdot x^{-\frac{1}{2}} \nu^{\frac{1}{6}}\left(\frac{1}{-\phi^{\prime}}\right)^{\frac{1}{2}}\left(\nu^{\frac{2}{3}} \phi\right)^{\frac{1}{2}} J_{1 / 3}\left(\frac{2}{3} \nu \phi^{\frac{3}{2}}\right) d r .
\end{aligned}
$$

It follows from (3) that,

$$
J_{1 / 3}\left(\frac{2}{3} \nu \phi^{\frac{3}{2}}\right)=\sigma_{1}\left(\nu \phi^{\frac{3}{2}}\right) e^{i \frac{2}{3} \nu \phi^{\frac{3}{2}}}+\sigma_{2}\left(\nu \phi^{\frac{3}{2}}\right) e^{-i \frac{2}{3} \nu \phi^{\frac{3}{2}}},
$$

where $\sigma_{i}$ is a symbol of order $-\frac{1}{2}$. We therefore write $\widetilde{A}=A_{1}+A_{2}$, where

$$
\begin{aligned}
A_{1}(\theta)= & 2^{j(\alpha-N \beta)} \int_{0}^{\infty} \eta_{3}(x) \vartheta(r) e^{i 2^{j \beta}\left[\varphi(r, \theta)+2^{-j \beta} \frac{2}{3} \nu \phi^{\left.\frac{3}{2}\right]}\right.}(x \nu)^{\frac{N+1-n}{2}} \\
& \cdot x^{-\frac{1}{2}} \nu^{\frac{1}{6}}\left(\frac{1}{-\phi^{\prime}}\right)^{\frac{1}{2}}\left(\nu^{\frac{2}{3}} \phi\right)^{\frac{1}{2}} \sigma_{1}\left(\nu \phi^{\frac{3}{2}}\right) d r \\
A_{2}(\theta)= & 2^{j(\alpha-N \beta)} \int_{0}^{\infty} \eta_{3}(x) \vartheta(r) e^{i 2^{j \beta}\left[\varphi(r, \theta)-2^{-j \beta \frac{2}{3} \nu \phi^{\left.\frac{3}{2}\right]}}(x \nu)^{\frac{N+1-n}{2}}\right.} \\
& \cdot x^{-\frac{1}{2}} \nu^{\frac{1}{6}}\left(\frac{1}{-\phi^{\prime}}\right)^{\frac{1}{2}}\left(\nu^{\frac{2}{3}} \phi\right)^{\frac{1}{2}} \sigma_{2}\left(\nu \phi^{\frac{3}{2}}\right) d r .
\end{aligned}
$$

Let us first consider the integral $A_{1}$, and now let $\widetilde{\Phi}_{\theta}(r)=\varphi(r, \theta)+2^{-j \beta} \frac{2}{3} \nu \phi^{\frac{3}{2}}$. We note that $\partial_{r} \widetilde{\Phi}_{\theta}(r)=\partial_{r} \Phi_{\theta}(r)$. It therefore follows that $\widetilde{\Phi}_{\theta}$ behaves exactly as $\Phi_{\theta}$ did in $\$ 5.2$, and so for $C_{\beta}$ chosen small enough we again wish to integrate by parts. In this case our amplitude

$$
\widetilde{\Psi}_{\theta}(r)=\eta_{3}(x) \vartheta(r)(x \nu)^{\frac{N+1-n}{2}} x^{-\frac{1}{2}} \nu^{\frac{1}{6}}\left(\frac{1}{-\phi^{\prime}}\right)^{\frac{1}{2}}\left(\nu^{\frac{2}{3}} \phi\right)^{\frac{1}{2}} \sigma_{1}\left(\nu \phi^{\frac{3}{2}}\right),
$$

courtesy of the symbol estimates $\left|\partial_{r}^{\ell} \sigma_{1}\left(\nu \phi^{\frac{3}{2}}\right)\right| \leq C x^{\frac{1}{4}+\ell}(\nu-x)^{-\frac{3}{4}-\ell}$, satisfies for $\ell=0,1, \ldots$ the differential inequality

$$
\left|\partial_{r}^{\ell} \widetilde{\Psi}_{\theta}(r)\right| \leq C(\nu-x)^{-\ell-\frac{1}{4}} \nu^{N-n+\frac{3}{4}+\ell} .
$$

Integrating by parts $N^{\prime}$ times we therefore get the estimate

$$
\begin{aligned}
\left|A_{1}(\theta)\right| & \leq C 2^{j(\alpha-N \beta)}\left|\int_{0}^{\infty} \widetilde{\Psi}_{\theta}(r) e^{i 2^{j \beta} \widetilde{\Phi}_{\theta}(r)} d r\right| \\
& \leq C 2^{j\left(\alpha-\left(N+N^{\prime}\right) \beta\right)} \int_{\nu^{\frac{1}{3}} \leq \nu-x}(\nu-x)^{-N^{\prime}-\frac{1}{4}} \nu^{N+N^{\prime}-\frac{1}{4}-n} d x \\
& \leq C 2^{j\left(\alpha-\left(N+N^{\prime}\right) \beta\right)} \nu^{N+\frac{2}{3} N^{\prime}-n} \\
& \leq C 2^{j\left(\alpha-n \beta-\frac{N^{\prime}}{3} \beta\right)},
\end{aligned}
$$

again provided $N>n$, but also that $N^{\prime} \geq 1$. We of course obtain the same estimate for $A_{2}$ since its phase is trivially never stationary. In Region $A$ we therefore obtain the estimate

$$
\left|\mu_{j, 3}\left(\kappa, \lambda 2^{2 j}\right)\right| \leq C 2^{j\left(\alpha-\left(n+\frac{1}{2}\right) \beta\right)} .
$$

Region $B: \lambda \geq C_{\beta} 2^{j \beta}$. Recall that $\lambda r^{2} \sin \theta \sim \nu$ and hence $\sin \theta \leq C \nu \lambda^{-1}$. Trivially we get the estimate $\left|\mu_{j}\left(\kappa, \lambda 2^{2 j}\right)\right| \leq C 2^{j \alpha} \lambda^{-n} \nu^{\frac{1}{2}}$; we'll use the oscillation to do better. Using the Airy asymptotic form (7) we may write $\mathcal{J}_{3}=c \mathcal{A}+\mathcal{E}_{A}$, where

$$
\mathcal{A}(r)=2^{j \alpha} \int_{0}^{\pi} \eta_{3}(x)(\sin \theta)^{n-1} e^{i \lambda r^{2} \cos \theta}(x \nu)^{\frac{1-n}{2}} x^{-\frac{1}{2}} \nu^{\frac{1}{6}}\left(\frac{1}{\phi^{\prime}}\right)^{\frac{1}{2}} \operatorname{Ai}\left(-\nu^{\frac{2}{3}} \phi\right) d \theta .
$$


ERROR TERM:

$$
\begin{aligned}
\int_{0}^{\infty} & \vartheta(r) r^{-1-\alpha}\left|\mathcal{E}_{A}(r)\right| d r \\
& \leq C 2^{j \alpha} \int_{0}^{\pi}(\sin \theta)^{n-1} \int_{\frac{1}{2}}^{2}(x \nu)^{\frac{1-n}{2}} x^{-\frac{3}{2}} \nu^{\frac{1}{6}}\left(\frac{1}{\phi^{\prime}}\right)^{\frac{1}{2}}\left|\widetilde{\mathrm{Ai}}\left(-\nu^{\frac{2}{3}} \phi\right)\right| d r d \theta \\
& \leq C 2^{j \alpha} \int_{0}^{\pi}(\sin \theta)^{n-1} \int_{\frac{1}{2}}^{2}(x \nu)^{-\frac{1}{2}\left(n+\frac{1}{3}\right)}\left(-\nu^{\frac{2}{3}} \phi\right)^{\frac{1}{2}}\left(-\nu \phi^{\frac{3}{2}}\right)^{-\frac{1}{2}} d r d \theta \\
& \leq C 2^{j \alpha} \int_{0}^{\pi}(\sin \theta)^{n-1} \int_{\frac{1}{2}}^{2}(x \nu)^{-\frac{1}{2}\left(n+\frac{1}{2}\right)} \nu^{\frac{1}{4}}(\nu-x)^{-\frac{1}{4}} d r d \theta \\
& \leq C 2^{j \alpha}(\lambda \nu)^{-\frac{1}{2}\left(n+\frac{1}{2}\right)} \int_{\nu}(\sin \theta)^{\frac{1}{2}\left(n-\frac{5}{2}\right)} \nu^{-\frac{3}{4}} \int_{\nu-x \leq \nu}(\nu-x)^{-\frac{1}{4}} d x d \theta \\
& \leq C 2^{j \alpha} \lambda^{-n} \nu^{-\frac{1}{2}} .
\end{aligned}
$$

Main term: As in Region $A$ it suffices to estimate

$$
\widetilde{\mathcal{A}}(r)=2^{j \alpha} \int_{0}^{\pi} \eta_{3}(x)(\sin \theta)^{n-1} e^{i \lambda r^{2} \cos \theta}(x \nu)^{\frac{1-n}{2}} x^{-\frac{1}{2}} \nu^{\frac{1}{6}}\left(\frac{1}{\phi^{\prime}}\right)^{\frac{1}{2}}\left(\nu^{\frac{2}{3}} \phi\right)^{\frac{1}{2}} J_{1 / 3}\left(\frac{2}{3} \nu \phi^{\frac{3}{2}}\right) d \theta,
$$

and since $\nu \phi^{\frac{3}{2}} \geq 1$ we shall, as before, write $\widetilde{\mathcal{A}}=\mathcal{A}_{1}+\mathcal{A}_{2}$, where

$$
\begin{aligned}
\mathcal{A}_{1}(r)= & 2^{j \alpha} \int_{0}^{\pi} \eta_{3}(x)(\sin \theta)^{n-1} e^{i\left[\lambda r^{2} \cos \theta+\frac{2}{3} \nu \phi^{\frac{3}{2}}\right]}(x \nu)^{\frac{1-n}{2}} \\
& \cdot x^{-\frac{1}{2}} \nu^{\frac{1}{6}}\left(\frac{1}{\phi^{\prime}}\right)^{\frac{1}{2}}\left(\nu^{\frac{2}{3}} \phi\right)^{\frac{1}{2}} \sigma_{1}\left(\nu \phi^{\frac{3}{2}}\right) d \theta, \\
\mathcal{A}_{2}(r)= & 2^{j \alpha} \int_{0}^{\pi} \eta_{3}(x)(\sin \theta)^{n-1} e^{i\left[\lambda r^{2} \cos \theta-\frac{2}{3} \nu \phi^{\frac{3}{2}}\right]}(x \nu)^{\frac{1-n}{2}} \\
& \cdot x^{-\frac{1}{2}} \nu^{\frac{1}{6}}\left(\frac{1}{\phi^{\prime}}\right)^{\frac{1}{2}}\left(\nu^{\frac{2}{3}} \phi\right)^{\frac{1}{2}} \sigma_{2}\left(\nu \phi^{\frac{3}{2}}\right) d \theta .
\end{aligned}
$$

Let us first consider the integral $\mathcal{A}_{1}$, and now let $\widetilde{\Phi}_{r}(\theta)=\lambda r^{2} \cos \theta+\frac{2}{3} \nu \phi^{\frac{3}{2}}$. As above we note that $\partial_{\theta} \widetilde{\Phi}_{r}(\theta)=\partial_{\theta} \Phi_{r}(\theta)$ and thus

$$
\partial_{\theta} \widetilde{\Phi}_{r}(\theta)=-\frac{1}{2} \lambda r^{2}\left(2 \sin \theta-\left(\frac{\nu-x}{x}\right)^{\frac{1}{2}} \cos \theta\right) .
$$

Therefore, since $\nu-x \leq x$ in this interval, it is easy to see that

$$
\left|\partial_{\theta} \widetilde{\Phi}_{r}(\theta)\right| \geq C \lambda
$$

provided $\cos \theta \leq \sin \theta$. With this in mind we shall this time write $\mathcal{A}_{1}=\mathcal{A}_{1}^{1}+\mathcal{A}_{1}^{2}$, where

$$
\begin{gathered}
\mathcal{A}_{1}^{1}(r)=2^{j \alpha} \int_{0}^{\pi}\left(1-\chi\left(\frac{1}{10} \tan \theta\right)\right) \widetilde{\Psi}_{r}(\theta) e^{i \widetilde{\Phi}_{r}(\theta)} d \theta \\
\mathcal{A}_{1}^{2}(r)=2^{j \alpha} \int_{0}^{\pi} \chi\left(\frac{1}{10} \tan \theta\right) \widetilde{\Psi}_{r}(\theta) e^{i \widetilde{\Phi}_{r}(\theta)} d \theta
\end{gathered}
$$

and

$$
\widetilde{\Psi}_{r}(\theta)=(\sin \theta)^{n-1}(x \nu)^{\frac{1-n}{2}} x^{-\frac{1}{2}} \nu^{\frac{1}{6}}\left(\frac{1}{\phi^{\prime}}\right)^{\frac{1}{2}}\left(\nu^{\frac{2}{3}} \phi\right)^{\frac{1}{2}} \sigma_{1}\left(\nu \phi^{\frac{3}{2}}\right) .
$$


Case 1: If $\tan \theta \geq 1$, then letting $\widetilde{\Psi}_{r}^{1}(\theta)=\left(1-\chi\left(\frac{1}{10} \tan \theta\right)\right) \widetilde{\Psi}_{r}(\theta)$ we see that

$$
\begin{gathered}
\left|\widetilde{\Psi}_{r}^{1}(\theta)\right| \leq C \nu^{\frac{3}{4}-n}(\nu-x)^{-\frac{1}{4}}, \\
\left|\partial_{\theta} \widetilde{\Psi}_{r}^{1}(\theta)\right| \leq C \nu^{\frac{7}{4}-n}(\nu-x)^{-\frac{5}{4}} .
\end{gathered}
$$

Letting $y=x\left(\lambda, r, \frac{\pi}{2}\right)$ we see that an application of van der Corput's lemma gives

$$
\begin{aligned}
\int_{0}^{\infty} & \vartheta(r) r^{-1-\alpha}\left|\mathcal{A}_{1}^{1}(r)\right| d r \\
& \leq C 2^{j \alpha} \lambda^{-1}\left(\int_{\frac{1}{2}}^{2}\left|\widetilde{\Psi}_{r}^{1}\left(\frac{\pi}{2}\right)\right| d r+\int_{0}^{\pi} \int_{\frac{1}{2}}^{2}\left|\partial_{\theta} \widetilde{\Psi}_{r}^{1}(\theta)\right| d r d \theta\right) \\
& \leq C 2^{j \alpha} \lambda^{-1} \nu^{-\frac{1}{4}-n}\left(\int_{\nu-y \leq \nu}(\nu-y)^{-\frac{1}{4}} d y+\nu \int_{0}^{\pi} \int_{\nu^{\frac{1}{3}} \leq \nu-x \leq \nu}(\nu-x)^{-\frac{5}{4}} d x d \theta\right) \\
& \leq C 2^{j \alpha} \lambda^{-1} \nu^{-\frac{1}{4}-n}\left(\nu^{\frac{3}{4}}+\nu^{\frac{11}{12}}\right) \\
& \leq C 2^{j \alpha} \lambda^{-\left(n+\frac{1}{3}\right)} .
\end{aligned}
$$

Here we have used the fact that $\nu \geq C \lambda$; this is a consequence of $\sin \theta \geq \frac{1}{2}$.

Case 2: If $\tan \theta \leq 1$, then letting $\widetilde{\Psi}_{r}^{2}(\theta)=\chi\left(\frac{1}{10} \tan \theta\right) \widetilde{\Psi}_{r}(\theta)$ we see that

$$
\begin{aligned}
\left|\widetilde{\Psi}_{r}^{2}(\theta)\right| \leq & C(\sin \theta)^{n-1} \nu^{\frac{3}{4}-n}(\nu-x)^{-\frac{1}{4}} \leq C \lambda^{-(n-1)} \nu^{-\frac{1}{4}}(\nu-x)^{-\frac{1}{4}}, \\
\left|\partial_{\theta} \widetilde{\Psi}_{r}^{2}(\theta)\right| \leq & C(\sin \theta)^{n-2} \nu^{\frac{3}{4}-n}(\nu-x)^{-\frac{1}{4}} \\
& +C(\sin \theta)^{n-1} \nu^{\frac{2}{3}-n}\left|\partial_{\theta}\left[\left(\nu^{\frac{2}{3}} \phi\right)^{\frac{1}{2}} \sigma_{1}\left(\nu \phi^{\frac{3}{2}}\right)\right]\right| \\
\leq & C \lambda^{-(n-2)} \nu^{-\frac{5}{4}}(\nu-x)^{-\frac{1}{4}}+C \lambda^{-(n-1)} \nu^{-\frac{1}{3}} \partial_{\theta}\left(\nu \phi^{\frac{3}{2}}\right)^{-\frac{1}{6}} .
\end{aligned}
$$

Here of course our phase may be stationary, however it is easy to see that

$$
\left|\partial_{\theta}^{2} \widetilde{\Phi}_{r}(\theta)\right| \geq C \lambda^{2}(\nu-x)^{-\frac{1}{2}} x^{-\frac{1}{2}} \geq \lambda^{2} \nu^{-1} .
$$

Arguing as above we see that van der Corput's lemma gives

$$
\begin{aligned}
\int_{0}^{\infty} \vartheta(r) r^{-1-\alpha}\left|\mathcal{A}_{1}^{2}(r)\right| d r & \leq C 2^{j \alpha} \lambda^{-1} \nu^{\frac{1}{2}} \int_{0}^{\pi} \int_{\frac{1}{2}}^{2}\left|\partial_{\theta} \widetilde{\Psi}_{r}^{2}(\theta)\right| d r d \theta \\
& \leq C 2^{j \alpha} \lambda^{-n}\left(\lambda \nu^{-1} \int_{\theta \leq \frac{\nu}{\lambda}} d \theta+\nu^{\frac{1}{6}} \int_{\frac{1}{2}}^{2} \int_{0}^{\pi} \partial_{\theta}\left(\nu \phi^{\frac{3}{2}}\right)^{-\frac{1}{6}} d \theta d r\right) \\
& \leq C 2^{j \alpha} \lambda^{-n}\left(\lambda \nu^{-1} \int_{\theta \leq \frac{\nu}{\lambda}} d \theta+\nu^{\frac{1}{4}} \int_{\frac{1}{2}}^{2}(\nu-y)^{-\frac{1}{4}} d r\right) \\
& \leq C 2^{j \alpha} \lambda^{-n}
\end{aligned}
$$

The integral $\mathcal{A}_{2}$ can be treated in exactly the same way, thus in Region $B$ we obtain

$$
\left|\mu_{j, 3}\left(\kappa, \lambda 2^{2 j}\right)\right| \leq C 2^{j \alpha} \lambda^{-n} .
$$

5.4. Neighborhood of the turning point: $|\nu-x| \leq \nu^{\frac{1}{3}}$. Here we just use a size estimate and the fact that $\left|\Lambda_{\kappa}^{\delta}(x)\right| \leq C \nu^{-\frac{1}{3}}$. This is the best we can do since $\nu \phi^{\frac{3}{2}} \leq \nu\left(\frac{\nu-x}{\nu}\right)^{\frac{3}{2}} \leq 1$. Notice again that $(\lambda \nu)^{\frac{1}{2}} \leq 2 \lambda$. 
Region $A: \lambda \leq C_{\beta} 2^{j \beta}$. We should integrate by parts and since $x \leq C(x \nu)^{\frac{1}{2}}$ it suffices to estimate

$$
\begin{aligned}
\left|I_{4}(\theta)\right| & =2^{j(\alpha-N \beta)}\left|\int_{0}^{\infty} \eta_{4}(x) \vartheta(r) e^{i 2^{j \beta} \varphi(r, \theta)}(x \nu)^{\frac{N+1-n}{2}} \Lambda_{\kappa-N}^{n-1+N}(x) d r\right| \\
& \leq C 2^{j(\alpha-N \beta)} \nu^{N-n} \int_{|\nu-x| \leq \nu^{\frac{1}{3}}} \nu^{-\frac{1}{3}} d x \\
& \leq C 2^{j(\alpha-n \beta)}
\end{aligned}
$$

provided $N>n$. So in Region $A$ we obtain the estimate

$$
\left|\mu_{j, 4}\left(\kappa, \lambda 2^{2 j}\right)\right| \leq C 2^{j(\alpha-n \beta)} .
$$

Region $B: \lambda \geq C_{\beta} 2^{j \beta}$. Here we shall not integrate by parts first, so we wish to estimate

$$
\begin{aligned}
\left|\mu_{j, 4}\left(\kappa, \lambda 2^{2 j}\right)\right| & =2^{j \alpha} \int_{0}^{\pi}(\sin \theta)^{n-1}\left|\int_{\frac{1}{2}}^{2} \eta_{4}(x) e^{i 2^{j \beta} \varphi(r, \theta)}(x \nu)^{\frac{1-n}{2}} \Lambda_{\kappa}^{n-1}(x) d r\right| d \theta \\
& \leq C 2^{j \alpha} \int_{\sin \theta \leq \frac{\nu}{\lambda}}(\sin \theta)^{n-1} \nu^{-n} \int_{|\nu-x| \leq \nu^{\frac{1}{3}}} \nu^{-\frac{1}{3}} d x d \theta \\
& \leq C 2^{\alpha} \lambda^{-n} .
\end{aligned}
$$

5.5. Monotonic region I: $\nu+\nu^{\frac{1}{3}} \leq x \leq \frac{3}{2} \nu$. Recall that here

$$
\left|\Lambda_{\kappa}^{\delta}(x)\right| \leq C \nu^{-\frac{1}{4}}(x-\nu)^{-\frac{1}{4}} e^{-\gamma_{1} \nu^{-\frac{1}{2}}(x-\nu)^{\frac{3}{2}}} .
$$

Notice that in this interval $(\lambda \nu)^{\frac{1}{2}} \leq \lambda$.

Region $A: \lambda \leq C_{\beta} 2^{j \beta}$. We should integrate by parts and since $x \leq C(x \nu)^{\frac{1}{2}}$ it suffices to estimate

$$
\begin{aligned}
\left|I_{5}(\theta)\right| & =2^{j(\alpha-N \beta)}\left|\int_{0}^{\infty} \eta_{5}(x) \vartheta(r) e^{i 2^{j \beta} \varphi(r, \theta)}(x \nu)^{\frac{N+1-n}{2}} \Lambda_{\kappa-N}^{n-1+N}(x) d r\right| \\
& \leq C 2^{j(\alpha-N \beta)} \nu^{N-n} \int_{x-\nu \geq \nu^{\frac{1}{3}}} \nu^{-\frac{1}{4}}(x-\nu)^{-\frac{1}{4}} e^{-\gamma_{1} \nu^{-\frac{1}{2}}(x-\nu)^{\frac{3}{2}}} d x \\
& \leq C 2^{j(\alpha-n \beta)} \int_{u \geq 1} u^{-\frac{1}{4}} e^{-\gamma_{1} u^{\frac{3}{2}}} d u \\
& \leq C 2^{j(\alpha-n \beta)}
\end{aligned}
$$

again provided $N$ is large enough. So we again in Region $A$ obtain the estimate

$$
\left|\mu_{j, 5}\left(\kappa, \lambda 2^{2 j}\right)\right| \leq C 2^{j(\alpha-n \beta)} .
$$


Region $B: \lambda \geq C_{\beta} 2^{j \beta}$. Here we shall not integrate by parts first, so we wish to estimate

$$
\begin{aligned}
& \left|\mu_{j, 5}\left(\kappa, \lambda 2^{2 j}\right)\right| \\
& \quad=2^{j \alpha} \int_{0}^{\pi}(\sin \theta)^{n-1}\left|\int_{\frac{1}{2}}^{2} \eta_{5}(x) e^{i 2^{j \beta} \varphi(r, \theta)}(x \nu)^{\frac{1-n}{2}} \Lambda_{\kappa}^{n-1}(x) d r\right| d \theta \\
& \quad \leq C 2^{j \alpha} \int_{\sin \theta \leq \frac{\nu}{\lambda}}(\sin \theta)^{n-1} \nu^{-n} \int_{x-\nu \geq \nu^{\frac{1}{3}}} \nu^{-\frac{1}{4}}(x-\nu)^{-\frac{1}{4}} e^{-\gamma_{1} \nu^{-\frac{1}{2}}(x-\nu)^{\frac{3}{2}}} d x d \theta \\
& \quad \leq C 2^{j \alpha} \int_{\sin \theta \leq \frac{\nu}{\lambda}}(\sin \theta)^{n-1} \nu^{-n} \int_{u \geq 1} u^{-\frac{1}{4}} e^{-\gamma_{1} u^{\frac{3}{2}}} d u d \theta \\
& \quad \leq C 2^{j \alpha} \lambda^{-n} .
\end{aligned}
$$

5.6. Monotonic region II: $x \geq \frac{3 \nu}{2}$. Here we have the trivial estimate $\left|\Lambda_{\kappa}^{\delta}(x)\right| \leq$ $C e^{-\gamma_{2} x}$. Again in this interval $(\lambda \nu)^{\frac{1}{2}} \leq \lambda$.

Region $A: \lambda \leq C_{\beta} 2^{j \beta}$. We should integrate by parts, and since $x \geq(x \nu)^{\frac{1}{2}}$ it suffices to estimate

$$
\begin{aligned}
\left|I_{6}(\theta)\right| & =2^{j(\alpha-N \beta)}\left|\int_{0}^{\infty} \eta_{6}(x) \vartheta(r) e^{i 2^{j \beta} \varphi(r, \theta)} x^{N}(x \nu)^{\frac{1-n}{2}} \Lambda_{\kappa-N}^{n-1+N}(x) d r\right| \\
& \leq C 2^{j(\alpha-N \beta)} \nu^{\frac{1-n}{2}} \int_{x \geq \nu} x^{N-1-\frac{n-1}{2}} e^{-\gamma_{2} x} d x \\
& \leq C 2^{j(\alpha-N \beta)}
\end{aligned}
$$

for all $N \geq 0$. So in Region $A$ we obtain the estimate

$$
\left|\mu_{j, 6}\left(\kappa, \lambda 2^{2 j}\right)\right| \leq C 2^{j(\alpha-N \beta)} .
$$

Region $B: \lambda \geq C_{\beta} 2^{j \beta}$. Here we shall not integrate by parts first, so we wish to estimate

$$
\begin{aligned}
\left|\mu_{j, 6}\left(\kappa, \lambda 2^{2 j}\right)\right| & =2^{j \alpha} \int_{0}^{\pi}(\sin \theta)^{n-1}\left|\int_{\frac{1}{2}}^{2} \eta_{6}(x) e^{i 2^{j \beta} \varphi(r, \theta)}(x \nu)^{\frac{1-n}{2}} \Lambda_{\kappa}^{n-1}(x) d r\right| d \theta \\
& \leq C 2^{j \alpha} \int_{0}^{\pi}(\sin \theta)^{n-1} \nu^{-n} \int_{x \geq \nu} e^{-\gamma_{2} x} d x d \theta \\
& \leq C 2^{j \alpha} \lambda^{-N} \nu^{-n} \int_{\sin \theta \geq \frac{\nu}{\lambda}}(\sin \theta)^{n-1-N} d \theta \\
& \leq C 2^{j \alpha} \lambda^{-n} \nu^{-N} .
\end{aligned}
$$

We have therefore established Theorem 5.1 .

\section{ACKNOWLEDGEMENTS}

This paper formed part of the author's Ph.D. thesis written at the University of Wisconsin at Madison. I would like to take this opportunity to thank my teacher and advisor Steve Wainger for his guidance. 


\section{REFERENCES}

1. R. Askey and S. Wainger, Mean convergence of expansions in Laguerre and Hermite series, American J. Math. 87 (1965), 695-708. MR0182834 (32:316)

2. A. Erdélyi, Asymptotic forms for Laguerre polynomials, J. Indian Math. Soc. 24 (1960), 235-250. MR0123751 (23:A1073)

3. C. Fefferman, Inequalities for strongly singular convolution operators, Acta Math. 124 (1970), 9-36. MR0257819 (41:2468)

4. C. Fefferman and E. M. Stein, $H^{p}$ spaces of several variables, Acta Math. 129 (1972), 137193. MR $0447953(56: 6263)$

5. C. L. Frenzen and R. Wong, Uniform asymptotic expansions of Laguerre polynomials, SIAM J. Math. Anal. 19 (1988), 1232-1248. MR957682 (89k:33012)

6. D. Geller, Fourier analysis on the Heisenberg group, Proc. Nat. Acad. Sci. U.S.A. 74 (1977), 1328-1331. MR0486312 (58:6069)

7. I. I. Hirschman, Multiplier Transforms I, Duke Math. J. 26 (1956), 222-242. MR0104973 $(21: 3721)$

8. N. Lyall, A class of strongly singular Radon transforms on the Heisenberg group, to appear in Proc. of Edin. Math. Soc.

9. E. M. Stein, Harmonic analysis: Real-variable methods, orthogonality, and oscillatory integrals, Princeton Univ. Press, Princeton, 1993. MR1232192 (95c:42002)

10. E. M. Stein and G. Weiss, Introduction to Fourier analysis on Euclidean spaces, Princeton Univ. Press, Princeton, 1971. MR0304972 (46:4102)

11. S. Thangavelu, Harmonic analysis on the Heisenberg group, Birkhäuser, Boston, 1998. MR:1633042 (99h:43001)

12. S. Wainger, Special trigonometric series in $k$ dimensions, Memoirs of the AMS 59, American Math. Soc., 1965. MR0182838(32:320)

13. T. H. Wolff, Lectures on harmonic analysis, University Lecture Series 29, American Math. Soc., 2003. MR2003254 (2004e:42002)

Department of Mathematics, University of Wisconsin, Madison, Wisconsin 53706

E-mail address: lyall@math.wisc.edu

Current address: Department of Mathematics, The University of Georgia, Boyd GSRC, Athens, Georgia 30602

E-mail address: lyall@math.uga.edu 